\title{
Lateral posterior parietal activity during reality monitoring discriminations of memories of high and low perceptual vividness
}

\author{
Danielle R. King ${ }^{1,2} \cdot$ Misty L. Schubert ${ }^{1} \cdot$ Michael B. Miller $^{1}$
}

Published online: 30 April 2015

(C) Psychonomic Society, Inc. 2015

\begin{abstract}
Regions of the lateral posterior parietal cortex (PPC) tend to be more active during recognition of previously studied items compared to correct rejection of unstudied items. Previously, we demonstrated that this effect is sourcespecific. While items that were encoded through visual perception elicited robust successful retrieval activity in the lateral PPC during a subsequent source memory test, items that were visually imagined did not elicit this effect. Memories of perceived events typically contain more perceptually-based contextual details than memories of imagined events. Therefore, source-based differences in lateral parietal activity might be due to a difference in the perceptual vividness of memories of perceived and imagined events. The goal of the present study was to test this hypothesis. Participants perceived and imagined items in both high and low perceptual vividness conditions. Experiment 1 demonstrated that memories for items encoded in the high vividness conditions contained significantly greater visual detail than memories encoded in the low vividness conditions. In Experiment 2, participants were scanned while they made source memory judgments about items that were previously perceived and imagined in high and low vividness conditions. Consistent with previous findings, the left lateral PPC was more active during retrieval of
\end{abstract}

Electronic supplementary material The online version of this article (doi:10.3758/s13415-015-0357-4) contains supplementary material, which is available to authorized users.

Danielle R. King

dking@utdallas.edu

1 Department of Psychological and Brain Sciences, University of California, Santa Barbara, CA, USA

2 Center for Vital Longevity and School of Behavioral and Brain Sciences, 1600 Viceroy Drive, Suite 800, Dallas, TX 75235, USA perceived compared to imagined events. However, lateral PPC activity did not vary according to vividness, suggesting that source effects in this region cannot be explained by a difference in the perceptual vividness of memories encoded through perception versus imagination.

Keywords Episodic memory · Inferior parietal · Parietal cortex $\cdot$ Recollection

Numerous studies have demonstrated that regions of the lateral posterior parietal cortex (PPC) are more active when subjects correctly endorse previously studied items as old compared to when they correctly reject new items (for reviews, see Cabeza, Ciaramelli, Olson, \& Moscovitch, 2008; Levy, 2012; Vilberg \& Rugg, 2008; Wagner, Shannon, Kahn, \& Buckner, 2005). This effect, which has been referred to as the parietal old/new effect, has been observed across a wide range of experimental stimuli and encoding and response conditions. In a recent study, we demonstrated that successful retrieval effects are source-specific (King \& Miller, 2014). Subjects perceived and imagined images of objects in response to a cue word, and then at test decided whether old and new cue words corresponded to items that were previously perceived, imagined, or new. The results revealed that regions of the left lateral PPC, in the vicinity of the intraparietal sulcus (IPS), were more active during recognition of previously perceived compared to imagined events. In addition, while perceived items elicited a robust, left-lateralized old/new effect in the parietal cortex, there was hardly any detectable effect associated with imagined events anywhere in the lateral posterior parietal cortex. These findings have important implications regarding theories of the functional contributions of the lateral PPC to recognition memory. The goal of the present study was to test whether these source differences can be explained by a 
difference in the qualitative nature of memories from perception and imagination.

Several theories have been proposed to account for parietal successful retrieval effects in the literature. For instance, attentional theories such as the attention to memory (AtoM) model (Cabeza et al., 2008; Ciaramelli, Grady, \& Moscovitch, 2008) and the attention reorienting hypothesis (Dobbins, Foley, Schacter, \& Wagner, 2002; Dobbins \& Wagner, 2005) suggest that regions of the lateral PPC contribute to recognition memory by directing or maintaining the allocation of attention to mnemonic information. Mnemonic accumulator models postulate that parietal regions play a role in integrating or accumulating memory-strength signals until a criterion is met resulting in an old/new recognition decision (Donaldson, Wheeler, \& Petersen, 2010; Hutchinson et al., 2014; Kahn, Davachi, \& Wagner, 2004; Wagner et al., 2005; Wheeler \& Buckner, 2003). Representational models, including the episodic buffer account (Vilberg \& Rugg, 2008) and the cortical binding of relational activity (CoBRA) hypothesis (Shimamura, 2011), suggest that parietal regions contribute to the integration or temporary representation of stored information that is represented in a form that is accessible to decision-making processes.

One distinguishing feature between the various models of parietal contributions to recognition memory involves whether the activations reflect mental representations of stored information (e.g., episodic buffer) or monitoring and control processes necessary for retrieval (e.g., attention to memory). It is becoming increasingly evident that distinct sub-regions of the lateral PPC contribute differentially to recognition memory. For instance, evidence suggests that more ventral aspects of lateral PPC are involved in the representation of stored information, rendering them sensitive to the amount or strength of the mnemonic signal (Guerin \& Miller, 2011; Shimamura, 2011; Vilberg \& Rugg, 2007, 2009a, b; although see Cabeza et al., 2008; Ciaramelli et al., 2008 for alternative explanations). In contrast, dorsal regions are thought to contribute to processes necessary for recognition memory. According to attentional theories, dorsal PPC is involved in directing top-down attention toward stored mnemonic information (Cabeza et al., 2008; Ciaramelli et al., 2008). Indeed, dorsal PPC is active under conditions of uncertainty and when more effortful retrieval is necessary (Cabeza et al., 2008; Kim \& Cabeza, 2007; Moritz, Gläscher, Sommer, Büchel, \& Braus, 2006). Other theories suggest that dorsal PPC accumulates mnemonic evidence (Donaldson et al., 2010). Recently, Hutchinson and colleagues (2014) suggested that dorsal PPC is functionally heterogenic, with SPL exhibiting top-down attentional control, and IPS accumulating mnemonic evidence in support of a recognition memory decision.

Although the precise mechanisms underlying parietal contributions to recognition memory remain uncertain, the consistency of observed effects across different stimuli and task designs suggest that this region's involvement in recognition memory goes beyond simple lower-level sensory or motor process. For instance, successful retrieval effects occur when the encoded stimuli are words (Donaldson, Petersen, \& Buckner, 2001; Henson, Hornberger, \& Rugg, 2005; Herron, Henson, \& Rugg, 2004; Kahn et al., 2004), faces (Guerin \& Miller, 2009; Leube, Erb, Grodd, Bartels, \& Kircher, 2003), pictures (Cansino, Maquet, Dolan, \& Rugg, 2002; Dobbins \& Wagner, 2005; Shannon \& Buckner, 2004; Slotnick, Moo, Segal, \& Hart Jr., 2003; Weis, Klaver, Reul, Elger, \& Fernández, 2004), natural sounds (Shannon \& Buckner, 2004), and non-verbal musical stimuli (Klostermann, Loui, \& Shimamura, 2009). Effects also occur across different response contingencies - when subjects are instructed to respond to both old and new items, old items only, or new items only (Shannon \& Buckner, 2004) suggesting that activation is not likely due to the planning or implementation of a motor response.

Given the ubiquity of successful retrieval effects in the literature, it was rather striking to find in our previous study (King \& Miller, 2014) that while items encoded through perception elicited a robust, left-lateralized old/new effect, items encoded through imagination were hardly associated with any successful retrieval response. Because of the rarity of reported cases for which one stimulus class does not evoke a parietal successful retrieval response while another class does, understanding why the lateral PPC appears to be source-specific will be important for refining theories regarding the role of this region in recognition memory. One plausible explanation is that source-based differences in activity were a result of an underlying difference in the quality or nature of memories of perceived and imagined events. Memories from perception and imagination have been shown to differ with respect to the relative amount of different types of qualitative details they contain. Specifically, memories derived through perception tend to contain more perceptual detail (Hashtroudi, Johnson, \& Chrosniak, 1990; Johnson, Raye, Foley, \& Kim, 1982; Johnson, Foley, Suengas, \& Raye, 1988; Lampinen, Odegard, \& Bullington, 2003; Schooler, Gerhard, \& Loftus, 1986; Suengas \& Johnson, 1988), whereas internally generated memories generally contain more information regarding the cognitive operations that were engaged during encoding (Johnson et al., 1988; Johnson, Raye, Foley, \& Foley, 1981). Thus, it is possible that the lateral PPC plays a specialized role in representing these perceptually-based contextual details of memories, and the difference in the perceptual vividness of memories of real and imagined events may be driving source-based differences in activity. Alternatively, the lateral PPC may truly be source-specific, playing a more prominent role in the representation of memories derived through perception than those generated internally. It is possible, for instance, that regions of the lateral PPC are involved in representing processes that were active during encoding, processes such as visual perception or imagination.

In the present study, we tested whether source-based differences in lateral PPC activity could be explained by a 
difference in the perceptual vividness of memories of perceived and imagined events. We asked subjects to perceive and imagine events under high and low perceptual vividness conditions. Prior to scanning, we ran a behavioral experiment (Experiment 1) to ensure that the perceptual vividness encoding manipulation resulted in memories that differed with respect to the amount of perceptually based contextual details they contained. Then, using functional magnetic resonance imaging (fMRI), we scanned subjects while they made source memory decisions about previously studied and new items (Experiment 2). If lateral PPC contributes to recognition memory by supporting the representation of perceptual details associated with episodic memories, then this region should exhibit effects of perceptual vividness as well as source. In addition, in contrast to previous findings, we should find evidence that this region is capable of representing memories of internally generated events, when they are rich in sensory detail. Alternatively, if the lateral PPC is truly source-specific, then we should not expect to find effects of perceptual vividness, nor should there be successful retrieval effects associated with imagined events, even when they are highly vivid.

\section{Experiment 1}

\section{Methods}

Participants Participants in Experiment 1 were 30 University of California, Santa Barbara undergraduates (17 female; aged 17-23 years, $M=18.6$ ) who participated for course credit in an Introductory Psychology course. All subjects gave informed consent according to the procedures approved by the UCSB Institutional Review Board.

Stimuli and apparatus Different experimental materials were used for each of the five encoding conditions (see Design and procedure). During encoding, on each trial either a high or low imageable word was presented. On trials where high imageable words were presented ( $n=240$ trials), either a black rectangle ( $n=80$ trials) or a photo of an object that corresponded to the cue word was presented which was either a color photo ( $n=80$ trials) or a blurred, black and white photo ( $n=80$ trials). On trials where low imageable words were presented ( $n=160$ trials), either a black line ( $n=80$ trials), or a short sentence that included the cue word ( $n=80$ trials) was presented. Photo and sentence stimuli presented during the encoding phase were randomly selected from sets of 320 color photos, 320 blurred photos, and 240 sentences. During the retrieval phase, only words were presented on each trial. All of the words from the encoding phase were presented along with an additional 80 high imageable and 80 low imageable new words.
All cue words were selected from the MRC psycholinguistics database (Wilson, 1988). From a list of 2460 words that met the original inclusion criteria (4-10 letters, 1-8 phonemes, and $1-7$ syllables) the 800 highest and 800 lowest imageable words were selected. To further narrow down the list of 800 low imageable words to the 240 words that were used in the experiment, we asked 16 undergraduate subjects to view a subset of the low imageable words and to try to generate sentences internally using those words. They were told to press one button on the keyboard when they successfully came up with a sentence using the word, or to press a different button if they were either unable to generate a sentence using the word or if they did not know the meaning of the word. The final list of 240 words selected for use in the experiment included words that subjects were able to generate sentences from at least $85 \%$ of the time in less than $2 \mathrm{~s}$ on average. The high imageable cue words used in the experiment were selected from the list of the 800 most highly imageable words. These words were selected so that they matched the low imageable words on both familiarity (high imageable words: $M=501.87, S D=68.36$; low imageable words: $M=504.78, S D=62.8$ ), and word length in letters (high imageable words: $M=5.55, S D=1.27$; low imageable words: $M=5.75, S D=1.16$ ). All cue words were presented in 48-point Helvetica font.

Short sentences were constructed using each of the low imageable words. Sentences ranged from 19-62 characters including spaces and punctuation $(M=39.93, S D=8.97)$, and were presented in 48-point Helvetica font. Photo stimuli were color photos of objects on a white background that corresponded to each of the highly imageable words. Images were resized to have a height of 300 pixels and widths that ranged from $97-800$ pixels $(M=329.66, S D=95.99)$. Blurry photo stimuli were constructed from the original photo stimuli and therefore also corresponded to each of the highly imageable words. In Adobe Photoshop, $85 \%$ monochromatic uniform noise was added to all of the images, which were then blurred with a 3.5 Gaussian pixel blur, and converted to black and white by setting the saturation to -100 . Stimuli were presented with the MATLAB Psychophysics Toolbox (www. psychtoolbox.org) on a MacBook Pro. Responses were made by pressing buttons on the keyboard and response and reaction time (RT) data were recorded in MATLAB.

Design and procedure The experiment was based on a $2 \times 3$, perceptual vividness (high (photo), low (sentence)) by source (perceive, imagine, new) within-subjects design, with one additional condition (perceive low vividness (blurry photo), see Fig. 1). The blurry photo condition served as a low vividness control condition to ensure that any differences in brain activity associated with the vividness manipulation were not simply due to a difference in stimulus type, i.e., perceiving and imagining photos versus perceiving and imagining sentences. Hence, we predicted that similar to items in the low sentence 


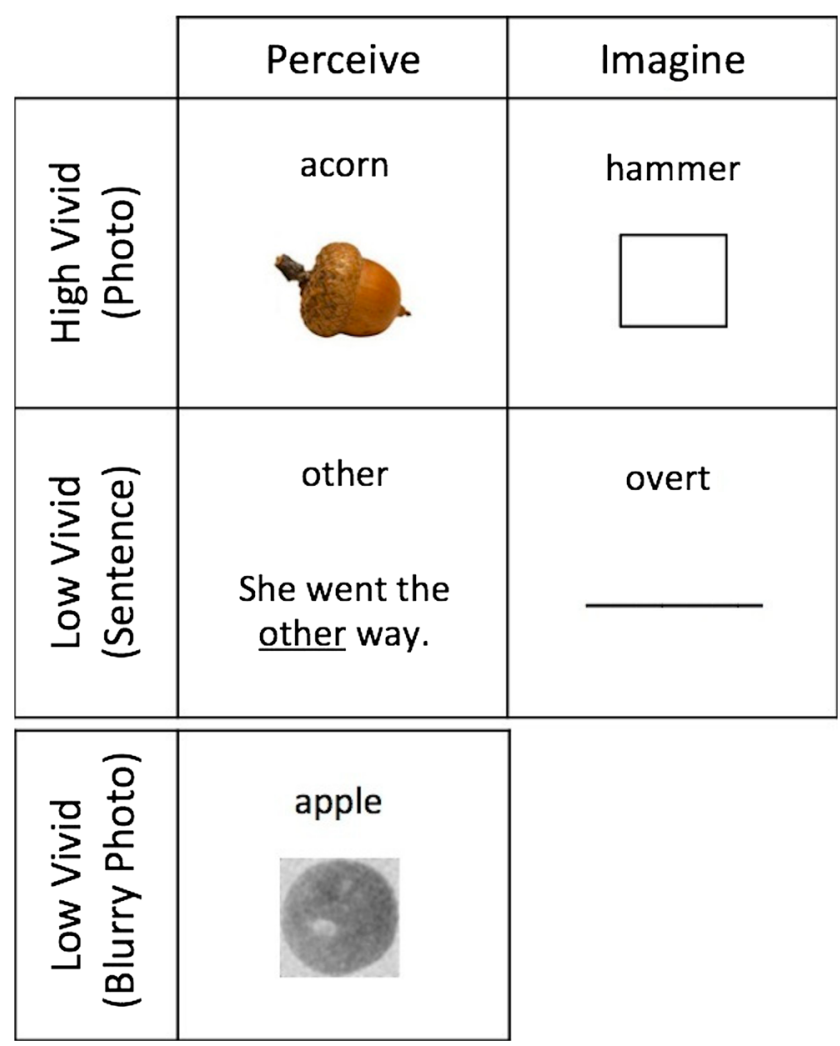

Fig. 1 Schematic representation of the five encoding conditions. Trials were separated into two photo and two sentence encoding blocks. Each photo block consisted of 40 high vividness (photo) perceive trials, 40 high vividness (photo) imagine trials, and 40 low vividness (blurry photo) perceive trials. Each sentence block consisted of 40 low vividness (sentence) perceive trials, and 40 low vividness (sentence) imagine trials. Within each block, trials from each condition were randomly intermixed. For each of the perceive conditions, subjects were instructed to encode both the cue word and the accompanying sentence or photo for a later memory test. For imagine conditions, subjects were instructed to try to generate an image (photo conditions) or sentence (sentence conditions) that corresponded with the cue word and try to remember both the word and generated image/sentence for a later memory test. Each trial lasted $3 \mathrm{~s}$, and the stimuli remained on the screen for the duration of the trial

perceive condition, memories of items encoded in the low blurry photo perceive condition would be rated as containing significantly less visual detail than items encoded in the high photo perceive condition. In terms of brain activity, regions that are sensitive to the degree of perceptual vividness of a retrieved memory should show greater activation during retrieval of items encoded in the high (photo) perceive condition than items encoded in both of the low perceive conditions (i.e., both the sentence and blurry photo conditions) if these effects are actually driven by differences in vividness and not stimulus type. We could not rely solely on the blurry photo condition for a fully counterbalanced design, as we found through piloting that individuals could not easily generate blurred, black and white images of objects (i.e., subjects reported automatically generating images of objects as they appear in the world and hence their imaginary experience did not differ from that of the high vividness imagine condition). Accordingly, we used low imageable words and had individuals perceive and imagine sentences rather than blurry photos in the low imageable conditions. However, we included the blurry photo perceive condition as a control to ensure that if there was a difference in parietal activity associated with the vividness manipulation that this difference could not be explained by the difference in stimulus type across the vividness conditions (i.e., high/low imageable words; pictures/sentence).

Experiment 1 involved both an encoding and a retrieval phase. Prior to encoding, participants were given a short practice run to help them acclimate to the pace of the experiment. The encoding phase consisted of two photo blocks and two sentence blocks. There were a total of $1203 \mathrm{~s}$ trials in each of the photo blocks, and $803 \mathrm{~s}$ trials in each of the sentence blocks. For each trial in the photo blocks, a highly imageable cue word was presented at the top of the screen. On high (photo) perceive trials ( $n=80$, or one-third of the photo block trials), a color image of an object corresponding to the cue word was presented in the center of the screen, below the cue word. For low (blurry photo) perceive trials $(n=80$, or one-third of the photo block trials), a blurred, black and white photo that corresponded to the cue word was presented, and for high (photo) imagine trials $(n=80$, or one-third of the photo block trials), a black rectangle, which served as a cue for subjects to visually imagine what the object looked like, was presented. Within each photo block, trials from each of the encoding conditions (photo perceive, blurry photo perceive, photo imagine) were randomly intermixed.

During each trial in the sentence blocks, a low imageable word was presented at the top of the screen. For low (sentence) perceive trials ( $n=80$, or one-half of the sentence block trials), a sentence containing the cue word was presented below the cue word. On low (sentence) imagine trials ( $n=80$, or onehalf of the sentence block trials), a black line was presented below the cue word, which served as a cue for subjects to generate a sentence in their head that contained the cue word.

Participants were instructed that on perceive trials, their task was simply to view the picture or read the sentence. For imagine photo trials, they were instructed to try to create rich, colorful, detailed images of objects similar to the photos of objects presented during perceive trials. For imagine sentence trials, participants were instructed to generate short, specific sentences that made use of the cue word (e.g., for the cue word "ran," instead of generating a generic sentence such as "I ran," participants were told to generate a more specific sentence, rendering it distinguishable from others, such as, "George ran to the park"). Throughout the encoding phase, subjects were instructed to encode both the cue word and the sentence/ photo that they perceived or imagined as best they could, but were not required to make any response. The order that photo and sentence blocks were presented was counterbalanced across subjects. The order for which stimuli appeared and the 
conditions they were assigned to (perceived, imagined, new) was randomly determined. The encoding phase consisted of a total of 400 trials, and took $20 \mathrm{~min}$ to complete.

Immediately following the encoding phase, subjects began the retrieval phase, which was broken into four experimental runs, involving 120 trials each. All of the cue words from the encoding phase along with 80 new high imageable words and 80 new low imageable words were randomly intermixed. On each trial, a cue word was presented and subjects were asked to characterize their memory associated with the cue word on a number of different scales. They first rated the likelihood that the cue word was old (studied) or new (not studied) on a scale of 1 (highly likely old) to 5 (highly likely new), where a 3 indicated an 'unsure' response. In the event of an old response (1-2), subjects responded to four additional questions. They first judged the likelihood that the item corresponding to the cue word was encoded as a photo $(1=$ highly likely photo $)$ or a sentence ( $5=$ highly likely sentence; $3=$ unsure). Next, they judged the likelihood that the item was encoded through perception $(1=$ highly likely perceived $)$ or imagination $(5=$ highly likely imagined; $3=$ unsure). They then rated their memory for the item corresponding to the cue word for how much visual detail it contained ranging from 1 (None) to 5 (A lot). And finally, subjects indicated the extent to which they could recall what they were thinking about at the time of encoding, ranging from 1 (None) to 5 (A lot). Subjects had unlimited time to respond to each question. For each question, they were shown a scale with number references as well as words serving as a reminder of which attribute they were judging and the response mapping (e.g., " $1=$ highly likely photo/ 3 = unsure/ 5 = highly likely sentence").

\section{Results}

The purpose of Experiment 1 was to test whether the experimental manipulation of perceptual vividness resulted in memories that differed in the amount of visual detail they contained. To test this, we compared vividness ratings of items that were correctly identified as old and were attributed to the correct perceive/imagine source (regardless of confidence rating) from each of the five encoding conditions (note: for trials to be included in this analysis it was not necessary that they be attributed to the correct photo/sentence source; however, photo/ sentence source accuracy was very high, so the vast majority of trials included in this analysis were also attributed to the correct photo/sentence source).We compared vividness ratings using a one-way, repeated measures analysis of variance (ANOVA). The results of the ANOVA indicated that encoding condition had a significant effect on vividness ratings $(F(4,116)$ $=35.38, p<.001$, see Fig. 2). Post-hoc analyses (Sidak corrected ( $\mathrm{c}=10$ comparisons) family-wise alpha level of .05 $($ alpha $=.0051))$ revealed that visual detail ratings for items
Visual Detail Ratings

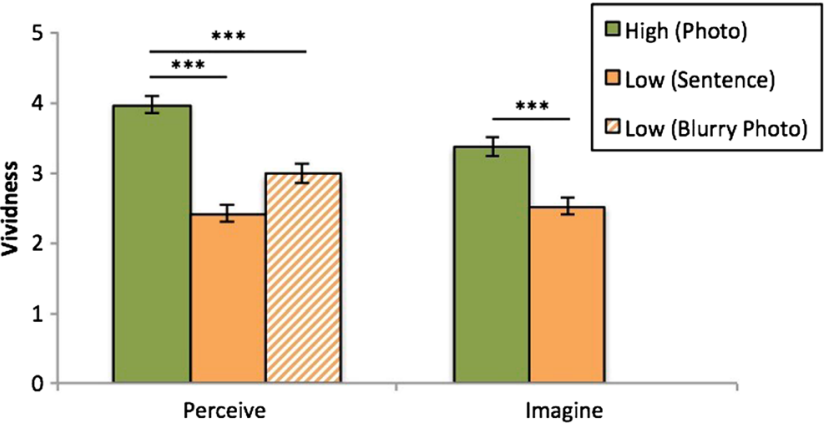

Fig. 2 Visual detail ratings from Experiment 1 across encoding conditions. During the test phase of Experiment 1, subjects rated their memory associated with each cue word for the amount of visual detail it contained on a scale of 1 (none) to 5 (a lot). Bars represent the mean vividness rating for items encoded in each of the five encoding conditions, and error bars are the standard error of the mean. Note that only the planned comparisons between vividness conditions within each source condition are denoted as significant here, although other pair-wise comparisons were significant as well. A description of all significant pair-wise tests is reported in the text. ${ }^{* * *} p<.001$

encoded in the high vividness (photo) perceive condition ( $M$ $=3.97, S D=0.74$ ) were significantly greater than vividness ratings of items encoded in each of the low vividness conditions (low (sentence) perceive: $M=2.41, S D=0.63, t(29)=9.08, p$ $<.001$; low (blurry photo) perceive: $M=2.98, S D=0.74, t(29)$ $=7.61, p<.001$; low (sentence) imagine: $M=2.52, S D=0.67$, $t(29)=8.02, p<.001)$. Similarly, items encoded in the high vividness (photo) imagine condition $(M=3.37, S D=0.74)$ were rated as containing significantly greater visual detail than items encoded in each of the low vividness conditions (low (sentence) perceive: $t(29)=5.81, p<.001$, low (blurry photo) perceive: $t(29)=3.49, p=.002$, and low (sentence) imagine: $t(29)=5.40, p<.001)$. In addition, visual detail ratings were significantly greater for items encoded in the high (photo) perceive condition compared to the high (photo) imagine condition $(t(29)=4.95, p<.001)$, and ratings were higher for items encoded in the low (blurry photo) perceive condition than the low (sentence) perceive condition $(t(29)=3.05, p=.005)$. Importantly, these results indicate that items encoded in each of the high vividness (photo) conditions were rated as containing significantly more visual detail than items encoded in the low vividness (sentence and blurry photo) conditions, for both perceived and imagined events.

We also analyzed vividness ratings according to a $2 \times 2$, source (perceive, imagine) by vividness (high (photo), low (sentence)), ANOVA, excluding the blurry photo perceive condition to test for main effects and interactions. The results revealed a significant source by vividness interaction $(F(1,29)$ $=41.44, p<.001$ ) as well as main effects of source (perceived $>$ imagined; $F(1,29)=60.21, p<.001$ ) and vividness (high (photo) $>$ low (sentence); $F(1,29)=10.21, p=.003$ ). The interaction was driven by a difference in the simple effects of source across vividness conditions, such that for items 
encoded in the high vividness (photo) condition, memories of perceived items were rated as containing significantly greater visual detail than memories of imagined events $(t(26)=9.08$, $p<.001$ ), whereas for items encoded in the low vividness (sentence) condition, vividness ratings did not differ according to source $(p>.05)$. However, importantly, for both perceived and imagined events, there was a significant simple effect of vividness, such that high vividness items were rated as containing more visual detail than low vividness items.

Behavioral performance in Experiment 1 was also assessed in terms of item memory, source memory, RT, and response criterion. Means and standard deviations for each of these measures according to encoding condition are presented in Table 1, and the results of statistical tests comparing means across conditions can be found in the Supplementary Material.

We also tested whether old/new confidence ratings differed according to encoding condition. The results of the ANOVA demonstrated that there was a significant effect of encoding condition on old/new confidence ratings of studied items recognized as old $(F(4,116)=10.79, p<.001)$. Old/new confidence was higher for items encoded in both the high (photo) perceive and low (sentence) perceive conditions relative to both high (photo) imagine $(t(29)=5.01, p<.001 ; t(29)=$ $4.14, p<.001$, respectively) and low (blurry photo) perceive conditions $(t(29)=3.15, p=.004 ; t(29)=3.05, p=.005$, respectively). We also analyzed confidence ratings according to a $2 \times 2$ source by vividness repeated measures ANOVA. The results demonstrated a significant interaction $(F(4,116)=$ $12.17, p<.001)$. For high (photo) hits, confidence was greater for perceived compared to imagined events; however, for low (sentence) hits, confidence did not vary according to source.

\section{Experiment 2}

\section{Methods}

Participants Thirty-five subjects (22 female; aged 2034 years, $M=26.4$ ) from the UCSB community volunteered to participate in response to an e-mail distributed to graduate students and staff. Subjects were right-handed, native English speakers with normal or corrected-to-normal vision. Data from eight subjects were excluded from analysis due to technical errors in data acquisition (two subjects), excessive motion ( $>3 \mathrm{~mm}$ of motion in a single direction in a single experimental run; three subjects), or an insufficient number of trials in any one (or more) of the seven conditions of interest (three subjects, $<15$ trials, conditions of interest were those that involved accurate source attributions of studied items from each of five encoding conditions as well as correct rejections of both high and low vividness new items, see Statistical modeling). All subjects gave informed consent according to the procedures approved by the University of California, Santa Barbara Institutional Review Board and were paid US\$60 for their participation.

Stimuli and apparatus Stimuli used in Experiment 2 were identical to those used in Experiment 1. Stimuli were again presented with the MATLAB Psychophysics Toolbox (www.psychtoolbox.org). During the encoding phase, which took place outside of the scanner, stimuli were presented on a MacBook Pro. Responses were made by pressing buttons on the keyboard, and response and RT data were recorded in MATLAB. During the retrieval phase, when subjects were scanned, images were projected from the MacBook Pro onto a screen situated at the head of the scanner, made visible to the participants by a mirror mounted to the head coil. The presentation of stimuli was synchronized with the onset of each functional scan in order to ensure accuracy of event timing. Behavioral responses and RTs were obtained by a fiber optic button box inside the scanner, and responses were recorded in MATLAB.

Design Just as in Experiment 1, Experiment 2 was based on a $2 \times 3$, perceptual vividness (photo, sentence) by source (perceive, imagine, new) within-subjects design, with one additional condition (perceived blurry photo).

Table 1 Means and (standard deviations) of behavioral measures from Experiments 1 and 2 across encoding conditions

\begin{tabular}{|c|c|c|c|c|c|c|c|c|}
\hline \multirow[t]{2}{*}{ Condition } & \multicolumn{2}{|l|}{ d' } & \multicolumn{2}{|l|}{ CSIM } & \multicolumn{2}{|l|}{$\mathrm{RT}$} & \multicolumn{2}{|l|}{$\mathrm{c}$} \\
\hline & Exp. 1 & Exp. 2 & Exp. 1 & Exp. 2 & Exp. 1 & Exp. 2 & Exp. 1 & Exp.2 \\
\hline High (photo) perceive & $1.81(0.76)$ & $1.76(0.66)$ & $0.90(0.09)$ & $0.89(0.08)$ & $1.01(0.44)$ & $1.50(0.18)$ & $0.23(0.43)$ & $-0.08(0.31)$ \\
\hline High (photo) imagine & $1.92(0.89)$ & $2.13(0.84)$ & $0.82(0.23)$ & $0.81(0.14)$ & $1.95(0.79)$ & $1.54(0.20)$ & $0.17(0.44)$ & $-0.27(0.28)$ \\
\hline Low (sentence) perceive & $1.23(0.51)$ & $1.27(0.57)$ & $0.70(0.19)$ & $0.69(0.18)$ & $2.06(0.84)$ & $1.76(0.19)$ & $0.46(0.59)$ & $0.03(0.35)$ \\
\hline Low (sentence) imagine & $1.80(0.79)$ & $1.81(0.58)$ & $0.80(0.25)$ & $0.76(0.15)$ & $2.12(0.64)$ & $1.74(0.19)$ & $0.17(0.62)$ & $-0.24(0.32)$ \\
\hline Low (blurry photo) perceive & $1.56(0.60)$ & $1.64(0.60)$ & $0.82(0.11)$ & $0.84(0.13)$ & $1.34(0.60)$ & $0.85(0.11)$ & $0.35(0.49)$ & $-0.02(0.31)$ \\
\hline
\end{tabular}

Notes: Item memory (d') assessed according to Signal Detection Theory, reality monitoring performance assessed according to the Conditional Source Identification Measure (CSIM), reaction time (RT) in seconds, and criterion (c) assessed according to Signal Detection Theory. RT is the average response latency to trials attributed to the correct perceived/imagined source 
Procedure The encoding phase of Experiment 2 was identical to that of Experiment 1. The retrieval phase was similar, with a few small exceptions. First, subjects were scanned during the retrieval phase of Experiment 2, so there was a delay of about 20 min between encoding and retrieval phases while subjects were situated in the scanner and while localizer and anatomical scans were collected. In addition, event trials were intermixed with jitter trials, included for statistical modeling purposes, which involved the presentation of a central fixation cross on the screen and did not require a response. The main difference between Experiments 1 and 2 was that subjects were not asked to make five judgments for every item and to scale their responses as in Experiment 1. Instead, subjects made only a single judgment regarding whether each item was either previously perceived, imagined, or new (regardless of whether it was perceived/imagined as a photo or a sentence). In addition, in Experiment 2, subjects had only $3 \mathrm{~s}$ to respond on each trial, whereas in Experiment 1 response time was unlimited.

Each retrieval block lasted $12 \mathrm{~min}, 45 \mathrm{~s}$ for a total functional scanning time of $51 \mathrm{~min}$. The entire scanning session lasted roughly $90 \mathrm{~min}$. The optimal sequence for the order of presentation of trials by condition was determined by randomly generating a series of event sequences $(n=1000)$ with the specified parameters (number of events, number of trials per event, trial length), creating design matrices from these sequences, and then identifying the design matrix with the smallest maximum eigenvalue of the inverse information matrix. A new sequence was generated for each subject, for each experimental run.

fMRI acquisition Imaging was performed at the UCSB Brain Imaging Center on a $3 \mathrm{~T}$ Siemens TIM Trio scanner with a standard 12-channel coil. Prior to functional scanning, an anatomical scan was collected using a magnetizationprepared rapid acquisition gradient-echo sequence $\left(\right.$ MPRAGE; TR $=2300 \mathrm{~ms} ; \mathrm{TE}=2.98 \mathrm{~ms} ; \mathrm{FA}=9^{\circ} ; 160$ saggital slices; $1.1 \mathrm{~mm}$ thick; $256 \times 256$ matrix). The four task-based functional runs included a series of $\mathrm{T} 2 *$-weighted whole-brain echoplanar images (EPI; $1500 \mathrm{~ms}$ repetition time (TR), $30 \mathrm{~ms}$ echo time (TE), $90^{\circ}$ flip angle). Each volume consisted of 28 slices acquired parallel to the AC-PC line (interleaved acquisition; $3 \mathrm{~mm}$ slice thickness, $64 \times 64$ matrix). Four volumes were discarded prior to the onset of each run to allow for tissue magnetization, followed by a series of 510 volumes.

Preprocessing Standard preprocessing was conducted using SPM8 (http://www.fil.ion.ucl.ac.uk/spm/). For each subject, time-series data were realigned to the mean functional image and sampled according to a seventh-degree B-spline interpolation method. Motion parameters were examined and used to exclude subjects with excessive motion $(>3 \mathrm{~mm}$ in one direction within an experimental run). Co-registration was performed as a three-step process, using a normalized mutual information cost function and 12-parameter affine transformations. First, the mean functional image was co-registered to the anatomical image. Next, the anatomical image was normalized to the SPM8 template image (MNI Avg152, T1 $2 \times 2 \times 2 \mathrm{~mm}$ ) and re-sampled with second-degree B-spline interpolation. Finally, the parameters from this transformation were used to register the functional images into MNI stereotaxic space. After normalization, data were spatially smoothed using an $8 \mathrm{~mm}$ full-width-half-maximum Gaussian kernel.

Statistical modeling Statistical Parametric Mapping (SPM8, Wellcome Department of Cognitive Neurology, London, UK) based on a General Linear Model (GLM) was used to analyze fMRI data. At the individual subject level, eight different events were modeled, which included seven events of interest with one additional event for all other trials (e.g., conditions of no interest, trials with multiple responses). The seven events of interest included correct responses to trials in each of the seven conditions (photo perceive trials called perceived; photo imagine trials called imagined; photo new trials called new; sentence perceive trials called perceived; sentence imagine trials called imagined; sentence new trials called new; and blurry photo perceive trials called perceived). The neural response elicited on each trial was modeled as a delta function corresponding to the onset of each trial. These functions were then convolved with a canonical hemodynamic response function to model the predicted BOLD response. An autoregressive AR(1) model used globally over the whole brain was applied during parameter estimation to correct for time-series correlations in the data.

At the individual subject level, contrast images were constructed that compared correct responses to old trials (hits) to correct responses to new trials (correct rejections or CRs) for each condition (photo perceive hits $>$ photo CRs; photo imagine hits > photo CRs; sentence perceive hits > sentence CRs; sentence imagine hits $>$ sentence CRs; blurry photo perceive hits $>$ photo CRs). The resulting hits $>$ CRs contrast images were then entered into two different second-level analyses. The hits $>$ CRs contrasts were entered into the second-level models because the cue words presented at retrieval differed according to perceptual vividness condition; the cue words for the photo conditions were highly imageable words whereas the cue words in the sentence conditions were low imageable words. To control for the imageability of words presented on the screen during the retrieval phase, we contrasted hits with correct rejections, which also varied with respect to imageability. The first second-level analysis tested for overall main effects of vividness and source. This analysis was based on a random effects, repeated measures factorial model $(2 \times 2$ : perceptual vividness (high (photo), low (sentence)) $\times$ source (perceive, imagine)), treating subjects as the random variable. 
The second analysis used a one-way, repeated measures analysis of variance (ANOVA) to examine successful retrieval effects associated with each of the five conditions of interest (high (photo) perceive, high (photo) imagine, low (sentence) perceive, low (sentence) imagine, and low (blurry photo) perceive). To account for within-subjects correlation of measures due to the repeated measure design, the covariance components were estimated with Restricted Maximum Likelihood (ReML) in SPM8 and used to adjust the statistics and degrees of freedom during inference.

Regions of interest analysis We ran several regions of interest (ROIs) analyses to examine the effects of perceptual vividness and source on lateral parietal activity. Regions were defined according to a study conducted by Nelson and colleagues (2010), in which resting state functional connectivity MRI (rs-fcMRI) boundary mapping was used to identify "correlationally" distinct regions in the left lateral PPC. In this study, $5 \mathrm{~mm}$ radius sphere ROIs were constructed at the center of 15 bounded regions within the left lateral PPC. These ROIs were then entered into a meta-analysis of recognition studies to identify which lateral PPC regions exhibited consistent successful retrieval effects. For the current analysis, we similarly constructed $5 \mathrm{~mm}$ radius sphere ROIs centered on the coordinates reported by Nelson and colleagues, and then extracted parameter estimates of successful retrieval activity associated with items encoded in each of the encoding conditions.

\section{Results}

Behavioral results Item memory, response bias, reality monitoring performance, and retrieval RT were compared across the five encoding conditions (Table 1). Item memory was assessed according to Signal Detection Theory (Green \& Swets, 1966), and sensitivity (d') and criterion (c) were calculated for each subject. Perceived and imagined responses were collapsed into one category of "old" responses, and, likewise, perceived and imagined trials were collapsed into a single category of old trials. Hence, hit rates reflected the proportion of perceived or imaged responses to all perceived and imagined items; similarly, the false alarm rate reflected the proportion of perceived or imagined responses to all new items. It is important to note that the current design did not allow for independent estimates of the false alarm rates associated with perceived and imagined events because at test, new items were randomly intermixed with perceived and imagined items. Hence, differences in either item memory (d') or criterion (c) according to source condition simply reflect a difference in the hit rates. Reality monitoring performance was assessed using the conditional source identification measure (CSIM; Murnane \& Bayen, 1996), which reflects the proportion of old items recognized as old that are attributed to the correct versus incorrect source. Average retrieval phase RTs were calculated for trials attributed to the correct source for each encoding condition.

A one-way, repeated measures ANOVA revealed that encoding condition had a significant effect on item memory $(F(4,104)=22.41, p<.001)$. Post-hoc comparisons were conducted using multiple paired-samples t-tests tested against a Sidak corrected (c = 10 comparisons) family-wise alpha level of .05 (alpha $=.0051)$. The results of the post-hoc comparisons revealed that item memory was significantly worse for items encoded in the low (sentence) perceive condition than all other conditions (high (photo) perceive: $t(26)=6.60, p$ $<.001$; high (photo) imagine: $t(26)=7.93, p<.001$; low (sentence) imagine: $t(26)=5.26, p<.001$; and low (blurry photo) perceive: $t(26)=4.92, p<.001)$. In addition, item memory was significantly better for items encoded in the high (photo) imagine condition than both the high (photo) perceive condition $(t(26)=5.72, p<.001)$ and the low (blurry photo) perceive condition $(t(26)=7.82, p<.001)$.

Reality monitoring also differed significantly depending on encoding condition $(F(4,104)=10.74, p<.001)$. Post-hoc analyses revealed that items encoded in the high (photo) perceive condition were attributed to the perceived/imagined source more accurately than items encoded in the low (sentence) perceive condition $(t(29)=6.57, p<.001$ ) and the low (sentence) imagine condition $(t(29)=4.38, p<$ $.001)$ ). Reality monitoring performance was also significantly better for items encoded in the low (blurry photo) perceive condition compared to the low (sentence) perceive condition $(t(29)=4.29, p<.001)$. There were no other differences in reality monitoring performance according to encoding condition. Overall, the behavioral results indicate that both item memory and source memory were worse for items encoded in the low (sentence) perceive condition compared to items encoded in most other conditions. In addition, item memory was slightly better for items encoded in the high (photo) imagine condition than other conditions, whereas reality monitoring performance was slightly better for items encoded in the high (photo) perceive condition than most other conditions.

Criterion differed significantly according to encoding condition $(F(4,104)=12.70, p<.001)$. Individuals were significantly more liberal when making judgments about items encoded in the high (photo) imagine condition than each of the perceive conditions (high (photo) perceive: $t(26)=5.71, p$ $<.001$; low (sentence) perceive: $t(26)=4.41, p<.001$; low (blurry photo) perceive: $t(26)=7.73, p<.001)$. In addition, individuals were more liberal when making judgments about items encoded in the low (sentence) imagine condition than the low (sentence) perceive $(t(26)=5.25, p<.001)$ and low (blurry photo) perceive conditions $(t(26)=3.91, p<.001)$. These results suggest that there individuals were more conservative in their judgments of perceived compared to imagined items, but there was no difference in criterion according to 
vividness condition. However, these results need to be interpreted with caution, as the current design did not allow for independent estimates of the false alarm rate associated with perceived and imagined events, and hence, the sourcebased criterion difference simply reflects a difference in the hit rates associated with perceived and imagined events.

Retrieval phase RTs associated with correctly attributed items varied according to encoding condition $(F(4,104)=$ $27.51, p<.001)$. RTs were slower for both the low (sentence) perceive and the low (sentence) imagine conditions than for all other conditions, which included the high (photo) perceive $(t(26)=7.33, p<.001 ; t(26)=6.59, p<.001$ respectively), the high (photo) imagine $(t(26)=5.19, p<.001 ; t(26)$ $=5.35, p<.001$ respectively), and the low (blurry photo) perceive condition $(t(26)=6.98, p<.001 ; t(26)=6.01, p<$ .001 , respectively). Hence, items encoded as photos were recognized faster than items encoded as sentences, but RTs did not differ according to perceived/imagined source.

To determine whether there was a bias to attribute items to either a perceived or imagined source, we further analyzed false alarms trials. We tested both whether individuals were biased toward responding either "perceived" or "imagined" to new test items, and whether this differed depending on the imageability of the test cue word. We tested this by comparing the proportion of "perceived" and "imagined" responses to high imageable and low imageable new words using a $2 \times 2$, source attribution by cue word imageability ANOVA. The results revealed a main effect of imageability $(F(1,26)=$ $14.78, p<.001)$ but no main effect of source attribution and no significant interaction. Overall, there was a higher false alarm rate to low imageable $(M=0.19, S D=0.12)$ than high imageable $(M=0.14, S D=0.12)$ words, but there was no difference in the tendency to attribute new items as "perceived" $(M=0.18, S D=0.11)$ versus "imagined" $(M=0.16, S D=0.12)$.

Whole-brain fMRI results To examine effects of vividness and source on successful retrieval activity, we ran two separate second-level, voxel-wise analyses on the whole-brain functional data. At the first level, hits (accurately attributed old items) from each of the encoding conditions were contrasted with correct rejections (see "Methods"). This resulted in five contrast images for each subject (high (photo) perceive hit $>$ high $\mathrm{CR}$, high (photo) imagine hit $>$ high $\mathrm{CR}$, low (sentence) perceive hit $>$ low $C R$, low (sentence) imagine hit $>$ low $C R$, low (blurry photo) hit $>$ high CR). These contrast images were then entered into two second-level, random effects analyses. The first analysis was based on a $2 \times 2$, repeated measures factorial model (source (perceived, imagined) by perceptual vividness (high (photo), low (sentence))). The purpose of this analysis was to determine whether there were main effects of source and perceptual vividness or if there was a source by vividness interaction. Therefore, the blurry photo perceive condition was not included in the analysis. However, the second analysis, which was conducted using a one-way, repeated measures ANOVA, identified successful retrieval activity associated with each of the five encoding conditions of interest. Contrast images constructed based on each of these secondlevel analyses were thresholded at $p<.001$, uncorrected, with a 23-voxel extent threshold (resulting in a cluster-wise corrected threshold of $p<.05$ according to the Monte-Carlo simulation implemented in AFNI; http://afni.nimh.nih.gov/ pub/dist/doc/program_help/AlphaSim.html). To locate anatomical regions, MNI coordinates were transformed into Talaraich space with the MatLab function mni2tal (http:// imaging.mrc-cbu.cam.ac.uk/imaging/MniTalairach) and entered into Talaraich Client software (Lancaster et al., 1997, 2000). All coordinates are reported in MNI $\{x, y, z\}$ stereotaxic space.

The $2 \times 2$ analysis of variance failed to identify any regions where there was a significant source by vividness interaction. When the threshold was dropped to $p<.005$, several regions were identified as showing an interaction; however, none of these regions were in the parietal cortex. There were, however, several regions that exhibited either a main effect of source or vividness on successful retrieval activity (Table 2, Fig. 3).

Main effect of source: With respect to source, recognition of perceived events was associated with greater activity than recognition of imagined events in several brain regions. The majority of voxels exhibiting this effect were in the parietal cortex. In the left hemisphere, activity was centered on the intraparietal sulcus (IPS), with the peak of activity in the inferior parietal lobe (IPL), extending to the supramarginal gyrus (SMG), angular gyrus (ANG), precuneus, and superior parietal lobe (SPL). In the right hemisphere, the peak of activity was again in the IPS, in a slightly more posterior region than in the left hemisphere. Activity extended to the SPL, ANG, IPL, and precuneus. A more medial parietal region also showed this source effect, with the peak of activity in the left precuneus, extending to the cuneus and SPL. Several prefrontal regions, with peaks in bilateral inferior frontal gyrus (IFG), left superior frontal gyrus (SFG), and right middle frontal gyrus (MFG) were also more active during recognition of perceived compared to imagined events. In addition, the left posterior cingulate cortex (PCC), left thalamus, and bilateral caudate were significantly more active during successful retrieval of perceived compared to imagined events. These results were consistent with our previous study (King \& Miller, 2014) in that a region of the lateral PPC, in the vicinity of the IPS, was more active during retrieval of perceived compared to imagined events. In contrast to the widespread activation elicited by the perceive $>$ imagine contrast, there were no brain areas that exhibited significantly greater activity during retrieval of imagined compared to perceived events.

Main effect of perceptual vividness: Next, we investigated which brain regions were sensitive to perceptual vividness by 
Table 2 Whole-brain main effects of source (perceive, imagine) and perceptual vividness (high (photo), low (sentence))

\begin{tabular}{llll}
\hline Region & Coordinates & Peak T & \# vox \\
\hline
\end{tabular}

Source by vividness interaction

None.

Perceive $>$ imagine

$\begin{array}{lrrrrr}\text { L IPS } & -39 & -58 & 52 & 5.21 & 349 \\ \text { R IPS } & 33 & -67 & 46 & 4.81 & 164 \\ \text { L Precuneus } & -9 & -70 & 43 & 4.72 & 121 \\ \text { L IFG } & -30 & 26 & -2 & 4.42 & 59 \\ \text { R IFG } & 33 & 29 & 4 & 4.13 & 39 \\ \text { L SFG } & -3 & 14 & 61 & 3.96 & 34 \\ \text { R MFG } & 51 & 29 & 25 & 3.92 & 24 \\ \text { L PCC } & -3 & -28 & 31 & 4.15 & 78 \\ \text { L Thalamus } & -9 & -19 & -2 & 4.50 & 165 \\ \text { R Caudate } & 12 & 8 & 13 & 4.41 & 78 \\ \text { L Caudate } & -12 & 2 & 10 & 4.02 & 41\end{array}$

Imagine $>$ perceive

None.

High (photo) $>$ low (sentence)

\begin{tabular}{lrrrrr} 
L MFG & -30 & 38 & -8 & 7.06 & 55 \\
R MFG & 30 & 38 & -11 & 5.75 & 70 \\
R IFG & 45 & 41 & 10 & 5.51 & 80 \\
L SOG & -39 & -85 & 31 & 6.29 & 105 \\
L ParaHipp. Gy. & -33 & -34 & -14 & 7.22 & 200 \\
R PCC & 6 & -37 & 37 & 4.35 & 104 \\
L PCC & -6 & -58 & 10 & 4.67 & 59 \\
R PCC & 6 & -52 & 10 & 4.29 & 39 \\
Low (sentence) $>$ high & $($ photo) & & & & \\
L IFG & -45 & 26 & -8 & 8.71 & 577 \\
L SFG & -6 & 14 & 61 & 4.86 & 115 \\
L MFG & -48 & 2 & 52 & 4.47 & 72 \\
R MOG & 36 & -85 & -5 & 4.25 & 31 \\
L STG & -54 & -49 & 7 & 6.45 & 245 \\
\hline
\end{tabular}

Notes: T-values and coordinates refer to the peak of the cluster, coordinates are in MNI stereotaxic space $\{\mathrm{x}, \mathrm{y}, \mathrm{z}\}$. Number of active voxels in cluster (\# vox); correct rejections (CR); left (L), right (R); angular gyrus (ANG); inferior frontal gyrus (IFG); inferior parietal lobe (IPL); middle frontal gyrus (MFG); middle occipital gyrus (MOG); parahippocampal gyrus (ParaHipp Gy.); posterior cingulate cortex (PCC); superior frontal gyrus (SFG); superior occipital gyrus (SOG); superior temporal gyrus (STG)

comparing activity associated with recognition of items encoded in the high (photo) versus low (sentence) perceptual vividness conditions (Table 2, Fig. 3). There were no voxels within the lateral PPC that demonstrated this vividness effect at an uncorrected threshold of $p<.001$ with a voxel extent of 23. This was true even at a more liberal threshold of $p<$ .005 , uncorrected, with a voxel extent of 23 . However, there were several other brain regions that were significantly more active during retrieval of items encoded in the high relative to the low perceptual vividness conditions. These included clusters of voxels with peaks in bilateral MFG, right IFG, left superior occipital gyrus (SOG), left parahippocampal gyrus, with activity extending to the hippocampus and amygdala, and bilateral PCC, with activity extending to the precuneus and cuneus.

The opposite contrast revealed several regions that were more active during retrieval of items encoded in the low vividness (sentence) compared to the high vividness (photo) conditions (Table 2, Fig. 3). The two largest clusters of voxels demonstrating this effect were in language/ semantic processing regions. The peak of activity within one of these clusters was in the left IFG or Broca's area (Broca, 1861; Tomaiuolo et al., 1999) and extended to the insula, the MFG, the middle and superior temporal gyri (MTG, STG), and the precentral gyrus. The other cluster was in the left posterior STG, or Wernicke's area (Wernicke, 1874). In addition, clusters of voxels with peaks in the left medial SFG, MFG, and right middle occipital gyrus (MOG), exhibited greater activity during recognition of items that were encoded as sentences relative to pictures.

To further investigate effects of vividness, we also examined the simple effect of vividness for perceived items only. If the lateral PPC simply does not exhibit successful retrieval activity associated with imagined events, then it is possible that activity in this region does vary according to perceptual vividness, but only for perceived events. Hence, vividness effects might have been masked in the main effect due to the inclusion of imagined events. However, the results of the simple effect contrast (high vividness (photo) perceive $>$ low vividness (sentence) perceive) were highly similar to the results of the main effect, with no significant differences in lateral PPC activity (neither at $p<.001$ or the reduced threshold of $p<.005$ ). This suggests that even when the analysis is restricted to perceived items, parietal successful retrieval activity does not vary according to perceptual vividness.

Successful retrieval effects: To further examine patterns of successful retrieval activity, we compared activity associated with correct source attributions of studied items (hits) to activity associated with correct rejection of new items separately for each of the five encoding conditions (Table 3, Fig. 4). Items encoded in the high (photo) perceive condition were associated with extensive successful retrieval activity. Regions demonstrating a successful retrieval effect included much of the lateral PPC, with the left hemisphere peak of activity in ANG extending into the IPL, SPL, SMG, precuneus, cuneus, SOG, MTG, STG, and PCC. In the right hemisphere, the peak of activity was in the ANG and activity extended to the IPL, SPL, SMG, and precuneus. Several areas in the PFC also exhibited successful retrieval effects for this condition. Peaks of activity were in right IFG, bilateral MFG, and right anterior medial SFG. In addition, clusters of voxels 


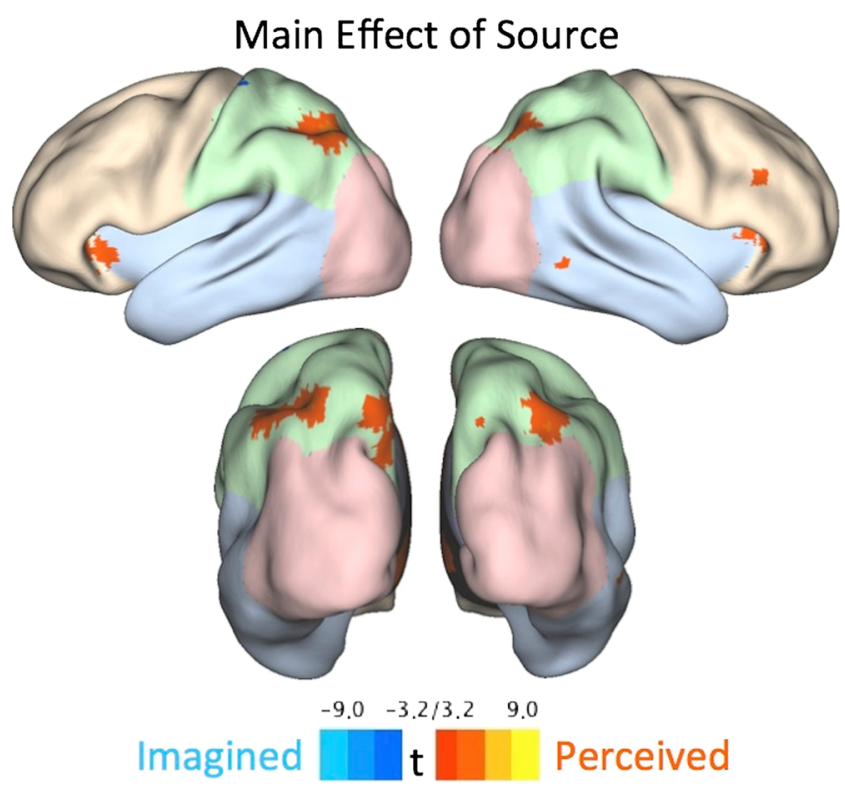

Fig. 3 Main effects of source and vividness. Images represent the grouplevel main effects of source (left) and vividness (right) from the wholebrain, voxel-wise analysis based on a $2 \times 2$ source (perceived, imagined) by vividness (high (photo), low (sentence)) ANOVA. Activations are mapped onto the inflated surface caret brain (Caret 5) and presented from

with peaks in both left and right MTG, left parahippocampal gyrus, lingual gyrus, PCC, bilateral caudate, and right putamen also exhibited greater activity during correct recognition of perceived events encoded in the high (photo) condition compared to correct rejections of highly imageable new items.

Compared to the high vividness (photo) perceive condition, successful retrieval activity associated with the low vividness (sentence) perceive condition was similar, only effects did not appear to be as widespread. In the left parietal cortex, the peak of activity was in the ANG, extending into the IPL, SPL, SMG, precuneus, MTG, and STG. In addition, successful retrieval effects were evident in left precuneus, extending into the SPL and PCC, as well as in the left IFG, SFG, and MTG, where activity extended to voxels in the left SMG.

Successful retrieval activity associated with the low (blurry photo) perceive condition was similar to the pattern of activity associated with the high (photo) perceive condition. In the left lateral PPC, activity peaked in the left IPL and extended to the SMG, SPL, ANG, MTG, and STG in the left hemisphere and the paracentral lobe, PCC, and precuneus bilaterally. In the right lateral PPC, activity peaked in the ANG, and extended to the IPL, SPL, SMG, precuneus, MTG, and STG. Successful retrieval effects were also evident in right IFG, bilateral MFG, right MTG, and left parahippocampal gyrus.

Compared to items that were perceived at encoding, successful recognition of imagined items was associated with far less activity. For the high vividness (photo) imagine condition, the only parietal regions exhibiting successful retrieval effects included a small cluster of 40 voxels with a peak of activity in left ANG. Successful retrieval effects were also

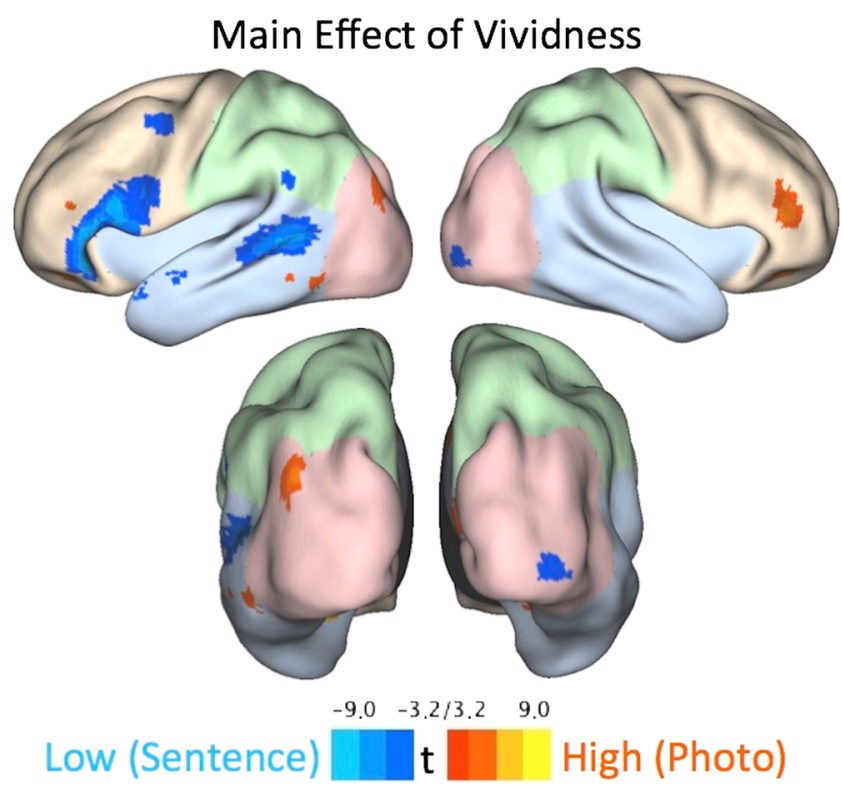

both the lateral (top) and posterior (bottom) views. The different lobes of the brain are represented in orange (frontal), green (parietal), pink (occipital), and blue (temporal). All voxels that exceeded the uncorrected threshold of $p<.001$ and are visible from the lateral or posterior views are displayed

evident in two different clusters in the left lateral PPC, both centered on the MFG.

For the low vividness (sentence) imagine condition, there was one small cluster of 24 voxels that demonstrated a successful retrieval effect in lateral PPC. This cluster was centered on the left SMG. Other regions that showed this effect were in left IFG, MFG, and STG. Overall, the results of the analysis of successful retrieval effects across different encoding conditions were consistent with our previous findings. Perceived events in both the high and low vividness conditions were associated with robust parietal successful retrieval activity, whereas imagined events elicited hardly any successful retrieval activity in lateral PPC.

Regions of interest analysis ROI analyses were conducted for 15 ROIs within the left lateral PPC defined according to Nelson et al. (2010; see "Methods", Fig. 5). We extracted parameter estimates of successful retrieval activity associated with perceived and imagined items encoded in the high and low perceptual vividness conditions. For each region, we conducted a $2 \times 2$, source (perceive, imagine) by vividness (high (photo), low (sentence)) repeated measures ANOVA to test for interactions and main effects (Fig. 6). To correct for multiple comparisons, $\mathrm{p}$-values were tested against a Sidak corrected alpha level (alpha of $p=.05$ was corrected for 15 comparisons, resulting in a corrected alpha level of $p=.0034$ ). The results of these analyses are presented in Figs. 5 and 6 and in Table 4.

Nelson and colleagues (2010) demonstrated that seven of 15 functionally distinct left lateral PPC regions exhibited reliable successful retrieval effects (Hit $>$ CR; see Fig. 5). 
Table 3 Whole-brain successful retrieval effects according to encoding condition

\begin{tabular}{|c|c|c|c|c|c|}
\hline Region & \multicolumn{3}{|c|}{ Coordinates } & Peak T & \# vox \\
\hline \multicolumn{6}{|c|}{ High (photo) perceive $>C R$} \\
\hline L ANG & -36 & -61 & 43 & 7.50 & 1460 \\
\hline R ANG & 39 & -64 & 49 & 6.18 & 578 \\
\hline R IFG & 51 & 41 & 10 & 6.55 & 292 \\
\hline L MFG & -27 & 41 & -11 & 7.27 & 752 \\
\hline R MFG & 24 & 35 & -14 & 5.18 & 52 \\
\hline R SFG & 15 & 62 & -11 & 3.75 & 24 \\
\hline L MTG & -60 & -52 & -11 & 6.45 & 101 \\
\hline R MTG & 60 & -49 & -11 & 5.26 & 76 \\
\hline L ParaHipp Gy. & -24 & -1 & -17 & 6.15 & 265 \\
\hline L Lingual Gyrus & -6 & -94 & -5 & 3.82 & 23 \\
\hline L PCC & -3 & -49 & 7 & 3.88 & 37 \\
\hline L Caudate & -9 & 5 & 4 & 3.45 & 29 \\
\hline R Caudate & 21 & -13 & 22 & 4.30 & 80 \\
\hline R Putamen & 21 & 2 & -11 & 4.14 & 25 \\
\hline \multicolumn{6}{|c|}{ Low (sentence) perceive $>C R$} \\
\hline L ANG & -48 & -64 & 52 & 5.99 & 486 \\
\hline L Precuneus & -6 & -70 & 43 & 5.47 & 221 \\
\hline L IFG & -51 & 26 & -2 & 7.59 & 1114 \\
\hline L SFG & -9 & 14 & 64 & 4.37 & 57 \\
\hline L MTG & -51 & -43 & 1 & 5.91 & 262 \\
\hline \multicolumn{6}{|c|}{ Low (blurry photo) perceive $>C R$} \\
\hline L IPL & -54 & -55 & 43 & 5.75 & 1293 \\
\hline R ANG & 42 & -64 & 52 & 5.61 & 587 \\
\hline R IFG & 51 & 41 & 10 & 5.43 & 151 \\
\hline L MFG & -42 & 20 & 49 & 5.78 & 617 \\
\hline R MFG & 42 & 11 & 55 & 4.58 & 143 \\
\hline R MTG & 66 & -49 & -5 & 4.44 & 58 \\
\hline L ParaHipp Gy. & -30 & -34 & -14 & 5.01 & 41 \\
\hline \multicolumn{6}{|c|}{ High (photo) imagine $>C R$} \\
\hline L ANG & -39 & -76 & 43 & 3.55 & 40 \\
\hline L MFG & -33 & 53 & -8 & 5.31 & 123 \\
\hline L MFG & -45 & 17 & 46 & 4.15 & 28 \\
\hline \multicolumn{6}{|c|}{ Low (sentence) imagine $>C R$} \\
\hline L SMG & -63 & -49 & 31 & 4.09 & 24 \\
\hline L IFG & -54 & 17 & 1 & 6.36 & 530 \\
\hline L MFG & -48 & 5 & 52 & 4.61 & 125 \\
\hline L STG & -57 & -52 & 7 & 4.43 & 80 \\
\hline
\end{tabular}

Notes: T-values and coordinates refer to the peak of the cluster, coordinates are in MNI stereotaxic space $\{x, y, z\}$. Number of active voxels in cluster (\# vox); correct rejections (CR); left (L), right (R); angular gyrus (ANG); inferior frontal gyrus (IFG); inferior parietal lobe (IPL); middle frontal gyrus (MFG); middle temporal gyrus (MTG); parahippocampal gyrus (ParaHipp Gy.); posterior cingulate cortex (PCC); superior frontal gyrus (SFG); superior temporal gyrus (STG)
Accordingly, we refer to these regions as "successful retrieval regions," with all other regions referred to simply as "other parietal regions." There was not a single successful retrieval region that demonstrated a main effect of vividness. However, four of seven successful retrieval regions demonstrated a main effect of source, such that successful retrieval activity was significantly greater for perceived compared to imagined events (Fig. 6a-c, g.). Regions exhibiting this effect included posterior IPS (Fig. 6a), posterior middle IPS (Fig. 6b), anterior middle IPS (Fig. 6c), and posterior IPL (Fig. 6g). The anterior IPL demonstrated a significant source by vividness interaction (Fig. 5e). A post-hoc analysis revealed that for the high (photo) condition, perceived items elicited greater successful retrieval activity than imagined items $(t(26)=5.29, p<.001)$, whereas for the low (sentence) condition, successful retrieval activity did not vary according to source $(p>.05)$. There were no significant main effects or interactions in either the anterior IPS (Fig. 5d) or the AnG (Fig. 5f).

In the remaining eight left lateral PPC regions that were not shown by Nelson et al. (2010) to demonstrate a successful retrieval effect, there was no evidence for any main effects of source or vividness (Fig. 5h-o). However, there was one region, the anterior IPL (Fig. 5j), that demonstrated a significant source $\times$ vividness interaction. This interaction was driven by the same pattern of results as the successful retrieval region that is also labeled anterior IPL (see above) with an effect of source (perceived $>$ imagined) for items encoded in the high vividness (photo; $t(26)=3.41, p=.002$ ) but not the low vividness (sentence) condition $(p>.05)$.

The findings from the ROI analysis are consistent with the whole-brain analysis, demonstrating that there were no parietal regions that exhibited an overall main effect of vividness. However, two regions did demonstrate a vividness effect for perceived, but not imagined events, which were both located in the anterior portion of the IPL. Further, source effects occurred in a subset of successful retrieval regions, which were located predominantly in the more posterior aspects of the IPS, on both the lateral and medial banks.

\section{Discussion}

The results of the present study demonstrated that sourcebased differences in lateral parietal activity cannot be explained by a difference in the perceptual vividness of memories of perceived and imagined events. Experiment 1 confirmed that encoding items in the high perceptual vividness conditions resulted in memories that contained significantly greater visual detail than items encoded in the low perceptual vividness conditions. Critically, the results from Experiment 2 demonstrated that successful retrieval activity in lateral PPC did not vary according to the perceptual vividness of retrieved memories. Consistent with previous 

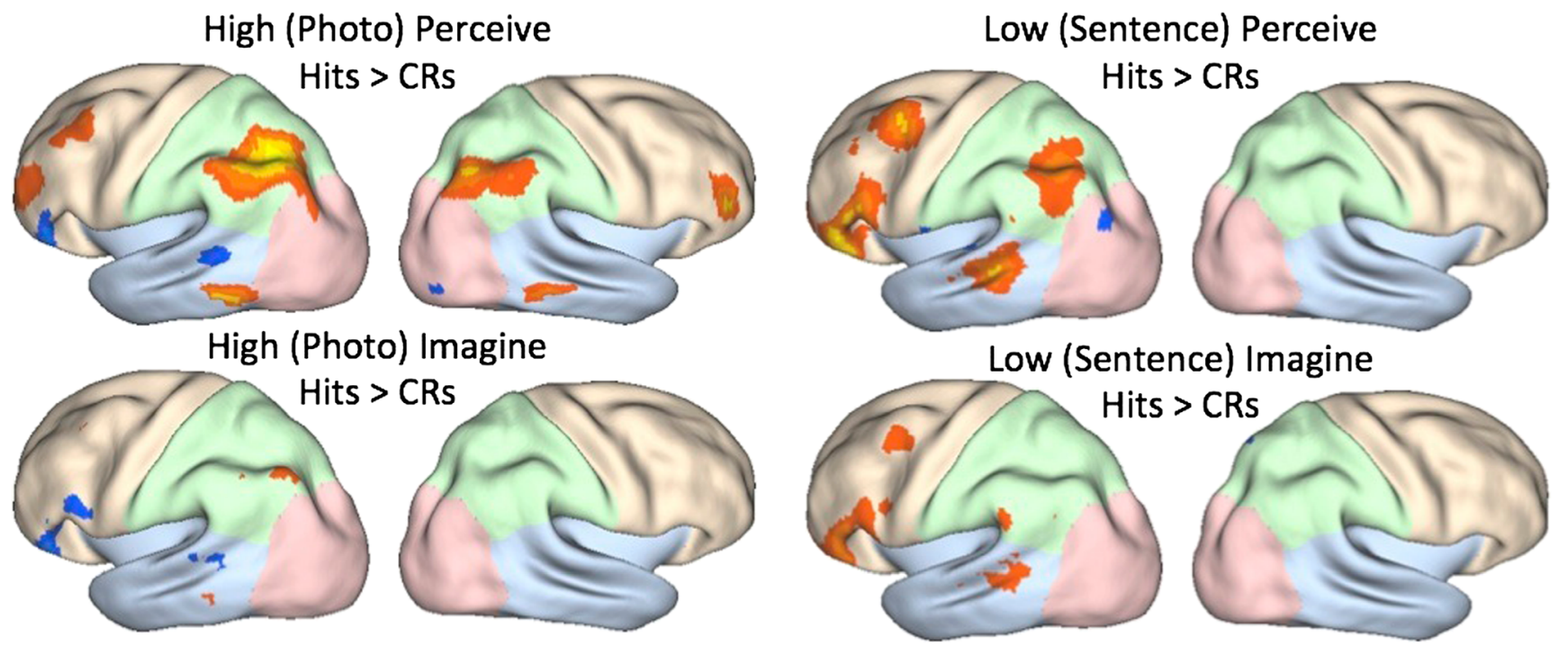

Low (Blurry Photo) Perceive

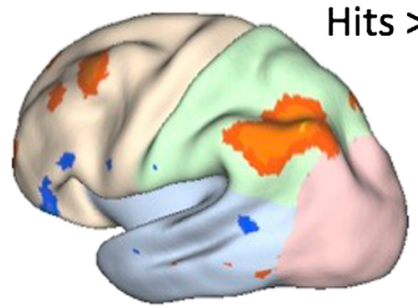

Fig. 4 Successful retrieval effects across encoding conditions. Brains represent successful retrieval activity (hits $>$ correct rejections (CRs)) associated with each of the five encoding conditions. Hits include items that were attributed to the correct source. Hits in the high (photo) perceive, high (photo) imagine, and low (blurry photo) perceive conditions were contrasted with correct rejections of high imageable new cue words, whereas hits in the low (sentence) perceive and low (sentence) imagine

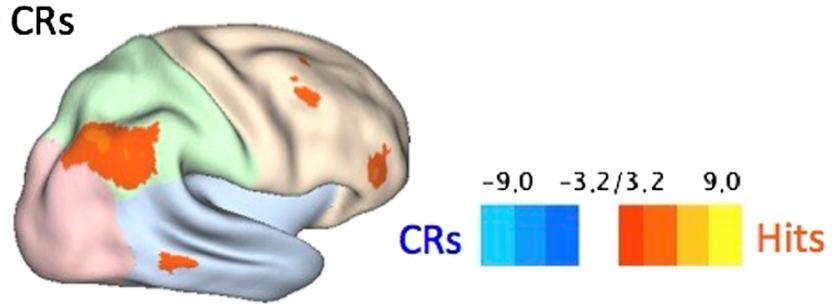

condition were contrasted with correct rejections of low imageable cue words. Activation maps are displayed on the inflated surface caret brain (Caret 5) and presented from the lateral view. The lobes of the brain are depicted in orange (frontal), green (parietal), pink (occipital), and blue (temporal). All voxels that exceeded the uncorrected threshold of $p<.001$ and are visible from the lateral view are displayed

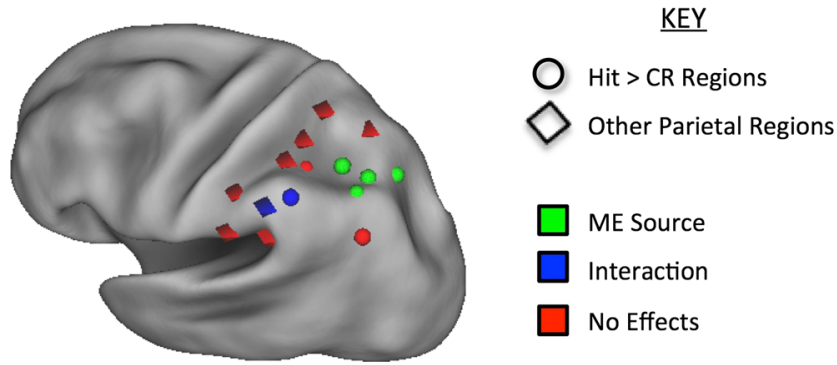

Fig. 5 Regions of interest defined according to Nelson et al. (2010). Peak coordinates of each region of interest are displayed on the inflated surface caret brain (Caret 5). Regions that were shown in a meta-analysis by Nelson et al. (2010) to demonstrate a successful retrieval effect (Hit > CR Regions) are represented by spheres (seven regions), whereas regions that did not demonstrate this effect (Other Parietal Regions) are displayed as diamonds (eight regions). Regions that demonstrated a main effect of source in the current study are presented in green (four regions); regions that demonstrated a significant source by vividness interaction are presented in blue (two regions); and regions where there was no main effect of source or vividness and no interaction are presented in red (nine regions; no region demonstrated a significant main effect of vividness; see Table 4 for statistics). Parameter estimates of successful retrieval activity for these regions are depicted in Fig. 6 findings (King \& Miller, 2014), we did find that regions of the lateral PPC were sensitive to source, with greater successful retrieval activity associated with previously perceived relative to previously imagined events. These results suggest that regions of the lateral PPC play a more prominent role in the representation of memories of real compared to imagined events, and this cannot be explained by a difference in the perceptual vividness of these memories.

Although activity in lateral PPC did not vary according to the perceptual vividness of recollected items, activity in several other regions did, suggesting that the failure to detect a vividness effect in lateral PPC was not simply due to insufficient power. For instance, regions in the ventral visual pathway were more active during retrieval of items encoded as photos compared to sentences. This activation likely reflects the reinstatement of encoding activity. Studies have shown that brain regions that are more active during encoding of one stimulus class over another show a similar pattern of differential activity during retrieval (Johnson \& Rugg, 2007; Kahn et al., 2004; Nyberg, Habib, McIntosh, \& Tulving, 
Fig. 6 Parameter estimates of successful retrieval activity associated with perceived and imagined events encoded in high and low vividness conditions in 15 left parietal regions of interest (ROIs). ROIs were defined as $5 \mathrm{~mm}$ radius spheres centered on the coordinates of 15 different left parietal regions defined according to a resting state parcellation conducted by Nelson and colleagues (2010). ROIs correspond to those depicted in Fig. 5. The y-axis represents the parameter estimate of successful retrieval activity (hits $>$ CRs) in arbitrary units. Significant main effects are denoted by a horizontal bar spanning across the two levels of a factor. For regions where there was a significant interaction, a horizontal bar above conditions corresponding to one level of a factor denote the presence of a simple effect, whereas 'n.s.' indicates a non-significant simple effect at that level of the factor. F and $p$-values of the statistical tests on these parameter estimates are presented in Table 4. (a) posterior intraparietal sulcus; (b) posterior middle intraparietal sulcus; (c) anterior middle intraparietal sulcus; (d) anterior intraparietal sulcus; (e) anterior inferior parietal lobe; (f) angular gyrus; (g) posterior inferior parietal lobe; (h) supramarginal gyrus; (i) anterior supramarginal gyrus; (j) anterior inferior parietal lobe; (k) lateral anterior intraparietal sulcus; (I) anterior intraparietal sulcus; (m) dorsal anterior intraparietal sulcus; (n) superior parietal lobe; and (o) dorsal superior parietal lobe
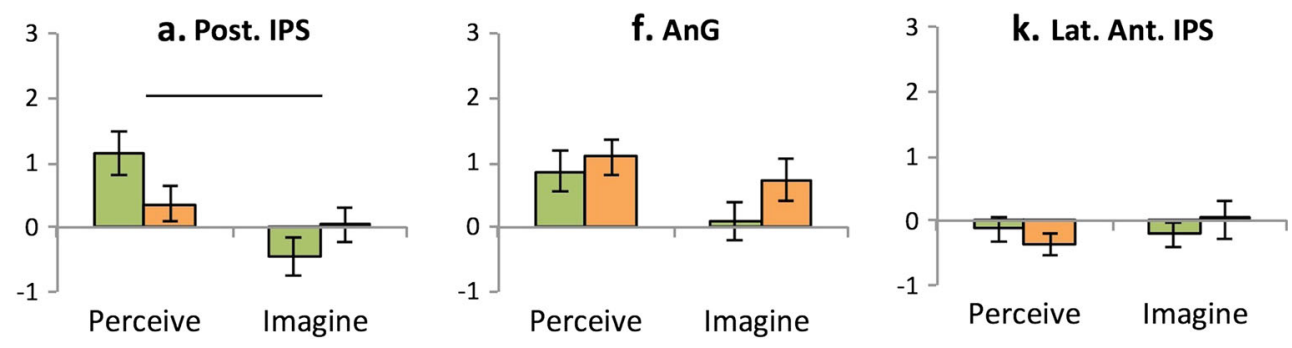

b. Post. Mid. IPS

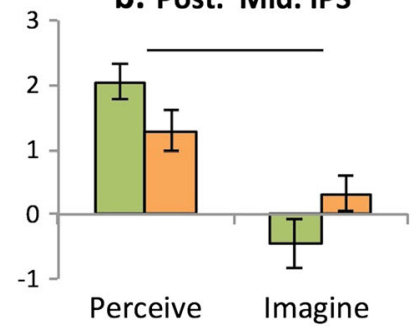

g. Post. IPL

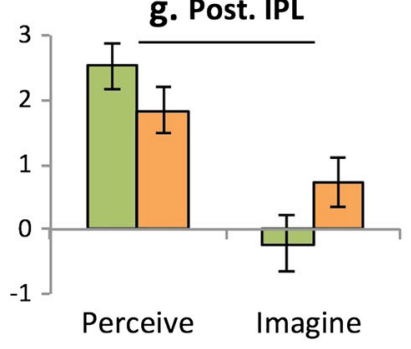

I. Ant. IPS
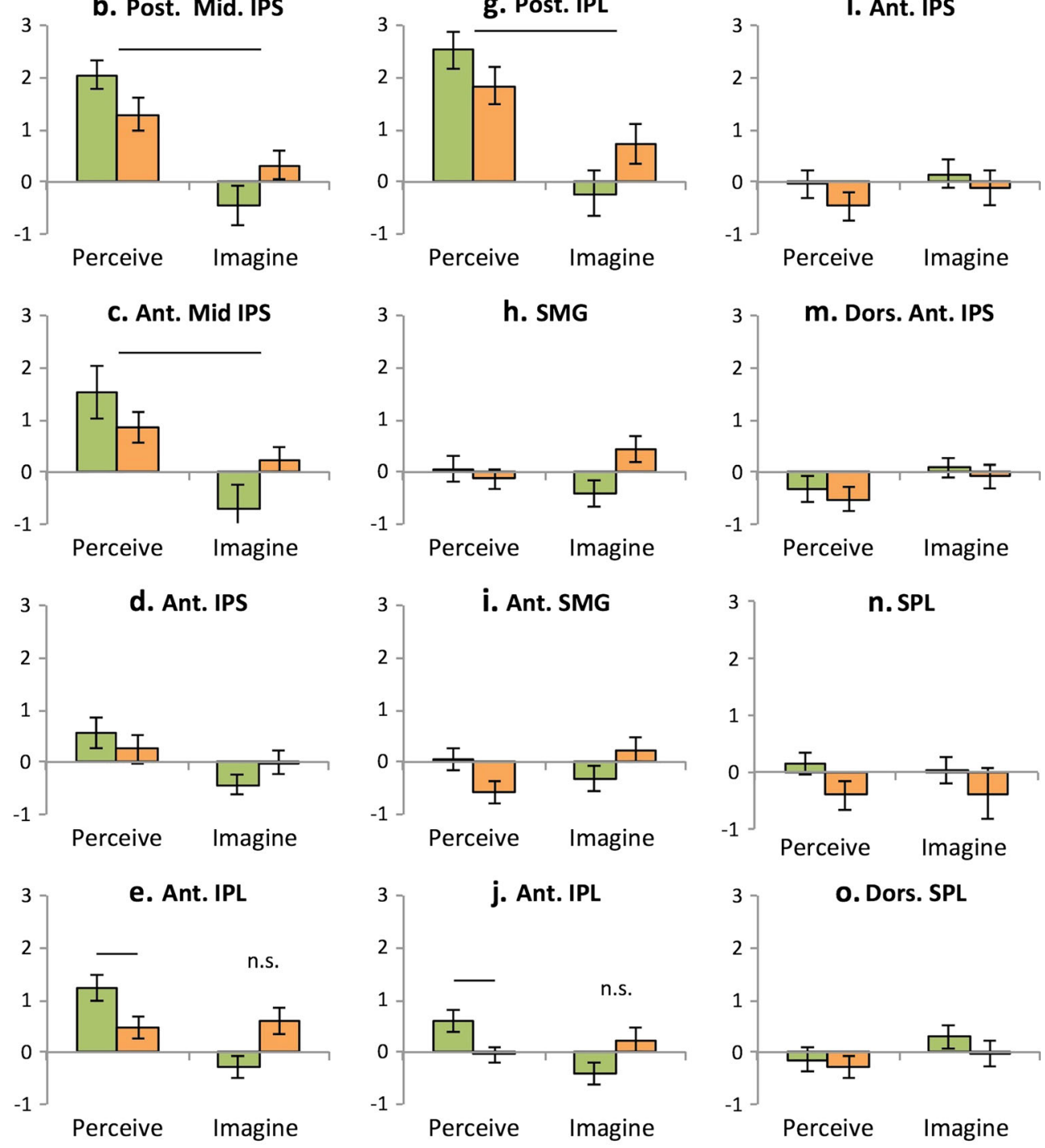

Low (Sentence)
2000; Vaidya, Zhao, Desmond, \& Gabrieli, 2002; Wheeler et al., 2006; Woodruff, Johnson, Uncapher, \& Rugg, 2005). Relevant to the current findings, studies have demonstrated that occipital regions that are more active during encoding of visual stimuli (e.g., objects, scenes) compared to verbal stimuli (e.g., words, sentences) show similar content-specific differences in activity during retrieval (Johnson \& Rugg, 2007; Vaidya et al., 2002). We also found that other brain regions, including language-processing regions, exhibited the opposite effect, with greater activity during source judgments of items that were encoded as sentences compared to photos. These regions included the left inferior frontal gyrus, or Broca's area (Broca, 1861; Tomaiuolo et al., 1999), as well as the left superior temporal gyrus, or Wernicke's area (Wernicke, 1874). These findings are again in line with a cortical reinstatement hypothesis. Prior studies have demonstrated that similar 
Table 4 Peak coordinates and statistics from the analysis of variance (ANOVA) that tested for effects of source (perceive, imagine) and vividness (high (photo), low (sentence)) on estimates of successful retrieval activity within 15 regions of interest (ROIs)

\begin{tabular}{|c|c|c|c|c|c|c|c|c|c|}
\hline & \multicolumn{3}{|c|}{ MNI coordinates } & \multicolumn{2}{|c|}{ ME vividness } & \multicolumn{2}{|c|}{ ME source } & \multicolumn{2}{|c|}{ Source $\times$ vivid interaction } \\
\hline & $\mathrm{x}$ & $\mathrm{y}$ & $\mathrm{z}$ & $F$ & $p$ & $F$ & $p$ & $F$ & $p$ \\
\hline \multicolumn{10}{|l|}{ Hits $>\mathrm{CR}$ regions } \\
\hline a. Post. IPS & -24 & -67 & 48 & 0.27 & 0.610 & 11.42 & 0.002 & 2.99 & 0.096 \\
\hline b. Post. Mid. IPS & -32 & -62 & 48 & 0.00 & 0.959 & 40.55 & $<.001$ & 4.91 & 0.036 \\
\hline c. Ant. Mid. IPS & -35 & -53 & 49 & 0.37 & 0.547 & 26.96 & $<.001$ & 7.23 & 0.012 \\
\hline d. Ant. IPS & -39 & -44 & 44 & 0.07 & 0.790 & 5.89 & 0.022 & 1.67 & 0.208 \\
\hline e. Ant. IPL & -54 & -47 & 48 & 0.05 & 0.828 & 10.10 & 0.004 & 18.21 & $<.001$ \\
\hline f. AnG & -45 & -67 & 36 & 1.64 & 0.211 & 2.62 & 0.118 & 0.82 & 0.373 \\
\hline g. Post. IPL & -40 & -62 & 48 & 0.25 & 0.620 & 27.78 & $<.001$ & 5.00 & 0.034 \\
\hline \multicolumn{10}{|l|}{ Other parietal regions } \\
\hline h. SMG & -56 & -42 & 31 & 2.61 & 0.118 & 0.03 & 0.859 & 4.83 & 0.037 \\
\hline i. Ant. SMG & -63 & -29 & 26 & 0.03 & 0.873 & 0.98 & 0.331 & 6.33 & 0.018 \\
\hline j. Ant. IPL & -60 & -40 & 44 & 0.00 & 0.969 & 2.78 & 0.108 & 12.47 & 0.002 \\
\hline k. Lat. Ant. IPS & -55 & -28 & 38 & 0.00 & 0.971 & 0.62 & 0.438 & 0.89 & 0.354 \\
\hline 1. Ant. IPS & -40 & -37 & 47 & 0.63 & 0.434 & 2.81 & 0.106 & 0.01 & 0.927 \\
\hline m. Dors. Ant. IPS & -36 & -41 & 54 & 1.07 & 0.311 & 0.81 & 0.376 & 0.07 & 0.790 \\
\hline n. SPL & -29 & -44 & 65 & 2.65 & 0.115 & 0.01 & 0.914 & 0.04 & 0.841 \\
\hline o. Dors. SPL & -24 & -58 & 62 & 1.35 & 0.256 & 2.31 & 0.141 & 0.10 & 0.753 \\
\hline
\end{tabular}

Notes: Regions were defined as 5-mm radius spheres centered on coordinates from Nelson et al. (2010). Here, regions are segregated according to whether Nelson et al. determined that regions demonstrated reliable successful retrieval effects (Hits $>$ CR regions) or not (Other parietal regions). Regions correspond to those depicted in Fig. 5. F and $p$ values from 15 separate ANOVA models that tested for main effects (ME) of vividness, source, and source by vividness interactions are presented. Tests that are significant at a Sidak corrected alpha level $(p<.05$ corrected for 15 comparisons, resulting in a corrected alpha level of $p<.0034$ ) are presented in bold font

regions tend to be more active during encoding and recollection of verbal compared to visual information (Johnson \& Rugg, 2007). Importantly, although cortical reinstatement is likely the mechanism underlying the content-specific vividness effects observed in the present study, it is unlikely that it can account for source effects, as activity in lateral PPC does not differ during visual perception compared to visual imagery (Ganis, Thompson, \& Kosslyn, 2004; King \& Miller, 2014; Kosslyn, Thompson, \& Alpert, 1997).

In the whole-brain analysis, regions that exhibited a main effect of source were predominantly in dorsal regions of the left lateral PPC, with activity centered on the IPS, extending dorsally to the SPL and ventrally to the IPL, including parts of the ANG. The results of the ROI analyses were consistent with the whole-brain findings. ROIs were defined according to a study in which the authors used rs-fcMRI boundary mapping in convergence with evidence from task-evoked studies to partition the left lateral PPC into functionally distinct regions (Nelson et al., 2010). The regions that demonstrated a source effect in the current study aligned closely with those labeled as left "intraparietal sulcus (LIPS)" and "posterior inferior parietal lobe (IPL)" by Nelson and colleagues (perceived > imagined; see Figs. 5 and 6, Table 4). In the context of recognition memory, the IPS has been implicated in a number of processes, most notably, in familiarity judgments (see Cabeza et al., 2008; Vilberg \& Rugg, 2008 for reviews). In addition, unlike other left lateral PPC regions, the IPS has been shown to exhibit successful retrieval activity in both recognition memory and cued recall tasks and during both explicit and implicit memory retrieval (Elman \& Shimamura, 2011; however, see Jaeger, Konkel, \& Dobbins, 2013). These studies suggest that the IPS plays a role in the processing of the 'oldness' of test items regardless of task demands. The findings from the current study suggest that this region is specifically involved in processing familiarity of perceived but not internally generated events. It does not seem likely that perceived events are simply more familiar than imagined events, as in the current study, item familiarity (as indexed by d') was actually worse for perceived than imagined events (although note the limitation of this measure of item memory within the context of the current study, see Experiment 2 - Behavioral results and below). Instead, these findings suggest that the IPS/ posterior IPL is specifically involved in representing memories of perceptually derived events, which may reflect a neural mechanism underlying our ability to make reality monitoring discriminations. The relative amount of activity within this region during retrieval may serve as a cue as to the perceived/ imagined source of a memory. 
These findings have implications for theories of parietal contributions to recognition memory. Representational models suggest that lateral parietal regions are involved in the online representation of features of memories during retrieval (Shimamura, 2011; Vilberg \& Rugg, 2008), whereas process models propose that lateral PPC contributes to processes that are necessary for retrieval such as orienting attention, accumulating mnemonic evidence, or post-retrieval evaluation (Cabeza et al., 2008; Ciaramelli et al., 2008; Donaldson et al., 2010; Hutchinson et al., 2014). The current findings are more consistent with a representational than a process model for the role of the IPS in recognition memory. This is not to suggest that the IPS is necessarily contributing to the representation of retrieved content, which has been suggested of more ventral regions in the lateral PPC, as this would likely manifest as differences in activity according to perceptual vividness. Instead, the IPS may play a role in representing information about the processes that were engaged during encoding, processes such as perceiving or imagining. However, it seems unlikely that source-based differences in activity are due to a difference in the processes engaged at retrieval. This is because source effects have been demonstrated regardless of differences in response accuracy and latency associated with perceived and imagined events, and across different encoding materials. Hence, it does not appear that there are inherent differences in the difficulty or task demands associated with retrieving memories of perceived versus imagined events. Therefore, the control or monitoring processes that are recruited during retrieval should not vary systematically depending on whether items were encoded through perception or imagination. While it is possible that the IPS is involved in representing other contextual features of memories, aside from perceptual details, the most likely explanation for source-based differences in IPS activity is that this region contributes to the representation of information about the processes that were engaged during encoding such as visual imagery or perception.

It should be noted that in the current study, the ratio of targets to lures differed across source conditions which could have contributed to the observed source-based differences in activity. However, this seems unlikely, as similar effects were detected in our previous study (King \& Miller, 2014), when the ratio of perceived and imagined events was equated across source conditions. Relatedly, response bias differed according to source condition, such that perceived items were associated with a more conservative response bias than imagined items. In light of recent evidence demonstrating that conservative responding can account for parietal successful retrieval activity (Aminoff et al., 2015), it is possible that the difference in criterion could account for source-based differences in parietal activity. However, as was noted previously, this difference in criterion, as measured here, simply reflects a difference in the hit rate, as independent estimates of the false alarm rates associated with each source condition were not possible. Although imagined trials were responded to more liberally, according to this measure of criterion, it is likely that the true false alarm rate for imagined items would be lower than for perceived items because of the mirror effect (Glanzer, Adams, Iverson, \& Kim, 1993; Glanzer \& Adams, 1985; Hirshman, 1995). Accordingly, imagined events might actually have been associated with a more conservative response bias than perceived events. If so, a response bias account would actually predict the opposite pattern of results, with greater activity associated with imagined than perceived events. Although the design of the current experiment is limited with respect to drawing inferences based on measures of decision criterion, it does not seem likely that a difference in response bias can account for source-based differences in parietal activity.

Although the source effects in left lateral PPC were highly consistent with our previous study, there was less consistency in other brain regions. For instance, in the present study, the pattern of activity in the right lateral PPC was similar to that of the left lateral PPC, with greater activity associated with recognition of perceived compared to imagined events. In our previous study, the source effect in the right lateral PPC was actually reversed. However, regions showing contrasting effects across the two studies were not anatomically analogous the right parietal source effect in our previous study was in a more anterior region of the parietal cortex than in the present study. It is still unclear why the lateral PPC was associated with the perceived $>$ imagined source effect bilaterally in the present study, and only in the left hemisphere in our past study. However, even in the present study, the source effect was stronger in the left hemisphere, and in general, memoryrelated activations in the parietal cortex tend to be leftlateralized (Guerin \& Miller, 2009).

Another region where the source effects were inconsistent across experiments was the left dorsolateral prefrontal cortex (DLPFC). Previously, this region showed evidence of a source effect, with greater activity during retrieval of perceived compared to imagined events, whereas in the present study, this effect was not replicated. However, when we examined source effects separately for items encoded in the photo and sentence conditions, we found that DLPFC did demonstrate a source effect in the photo, but not the sentence condition. Given that the stimuli presented in the photo condition in the present study were highly similar to those of our previous study, this suggests that source effects in left DLPFC might be materialspecific, with enhanced activity associated with retrieval of perceived versus imagined pictures, but not sentences. This is in contrast to the left lateral PPC which demonstrated a source effect for both photo and sentence stimuli.

To conclude, the results of the current study replicated previous findings (King \& Miller, 2014) demonstrating that recognition-related activity in the lateral PPC, specifically in 
the vicinity of the IPS, is modulated by internal/external source, with greater activity associated with accurate source discriminations of previously perceived compared to imagined events. The results of the present study also suggest that these source effects are not due to a difference in the perceptual vividness of memories from perception and imagination, as activity in lateral PPC did not vary according to the vividness of retrieved memories. The current study extended previous findings demonstrating that source effects in IPS occur regardless of differences in item and source memory accuracy associated with perceived relative to imagined events, and for both verbal and visual encoding materials. Whether the information represented in the IPS reflects information about the processes that were engaged during encoding or content remains to be determined, but given that source effects occurred regardless of differences in behavioral performance, these findings suggest a representational role for the IPS in recognition memory. These findings have important implications for theories of parietal contributions to recognition memory, and should be taken into consideration when refining extant models.

Acknowledgments This research was supported by the Institute for Collaborative Biotechnologies through grant W911NF-09-0001 from the US Army Research Office. The content of the information does not necessarily reflect the position or the policy of the Government, and no official endorsement should be inferred.

\section{References}

Aminoff, E. M., Freeman, S., Clewett, D., Tipper, C., Frithsen, A., Johnson, A., ... Miller, M. B. (2015). Maintaining a cautious state of mind during a recognition test: A large-scale fMRI study. Neuropsychologia, 67, 132-147. doi:10.1016/j.neuropsychologia. 2014.12.011

Broca, P. (1861). Perte de la parole, ramollissement chronique et destruction partielle du lobe antérieur gauche du cerveau. Bulletins de La Societe D'anthropologie, 2, 235-238.

Cabeza, R., Ciaramelli, E., Olson, I. R., \& Moscovitch, M. (2008). Parietal cortex and episodic memory: An attentional account. Nature Reviews Neuroscience, 9(8), 613-625. doi:10.1038/nrn2459

Cansino, S., Maquet, P., Dolan, R. J., \& Rugg, M. D. (2002). Brain activity underlying encoding and retrieval of source memory. Cerebral Cortex, 12(10), 1048-1056. doi:10.1093/cercor/12.10.1048

Ciaramelli, E., Grady, C. L., \& Moscovitch, M. (2008). Top-down and bottom-up attention to memory: A hypothesis (AtoM) on the role of the posterior parietal cortex in memory retrieval. Neuropsychologia, 46(7), 1828-1851. doi:10.1016/j.neuropsychologia.2008.03.022

Dobbins, I. G., \& Wagner, A. D. (2005). Domain-general and domainsensitive prefrontal mechanisms for recollecting events and detecting novelty. Cerebral Cortex, 15(11), 1768-1778. doi:10.1093/ cercor/bhi054

Dobbins, I. G., Foley, H. Schacter, D.L., \& Wagner, A. D. (2002). Executive control during episodic retrieval. Multiple prefrontal processes subserve source memory. Neuron, 35(5), 989-996.

Donaldson, D. I., Petersen, S. E., \& Buckner, R. L. (2001). Dissociating memory retrieval processes using fMRI: Evidence that priming does not support recognition memory. Neuron, 31(6), 1047-1059. doi:10. 1016/S0896-6273(01)00429-9

Donaldson, D. I., Wheeler, M. E., \& Petersen, S. E. (2010). Remember the source: Dissociating frontal and parietal contributions to episodic memory. Journal of Cognitive Neuroscience, 22(2), 377-391. doi: 10.1162/jocn.2009.21242

Elman, J. A., \& Shimamura, A. P. (2011). Task relevance modulates successful retrieval effects during explicit and implicit memory tests. NeuroImage, 56(1), 345-353. doi:10.1016/j.neuroimage.2011.02. 017

Ganis, G., Thompson, W. L., \& Kosslyn, S. M. (2004). Brain areas underlying visual mental imagery and visual perception: An fMRI study. Cognitive Brain Research, 20(2), 226-241. doi:10.1016/j. cogbrainres.2004.02.012

Glanzer, M., \& Adams, J. K. (1985). The mirror effect in recognition memory. Memory \& Cognition, 13(1), 8-20. doi:10.3758/ BF03198438

Glanzer, M., Adams, J. K., Iverson, G. J., \& Kim, K. (1993). The regularities of recognition memory. Psychological Review, 100(3), 546567.

Green, D. M., \& Swets, J. A. (1966). Signal detection theory and psychophysics. New York: Wiley.

Guerin, S. A., \& Miller, M. B. (2009). Lateralization of the parietal old/ new effect: An event-related fMRI study comparing recognition memory for words and faces. NeuroImage, 44(1), 232-242. doi: 10.1016/j.neuroimage.2008.08.035

Guerin, S. A., \& Miller, M. B. (2011). Parietal cortex tracks the amount of information retrieved even when it is not the basis of a memory decision. NeuroImage, 55(2), 801-807. doi:10.1016/j.neuroimage. 2010.11.066

Hashtroudi, S., Johnson, M. K., \& Chrosniak, L. D. (1990). Aging and qualitative characteristics of memories for perceived and imagined complex events. Psychology and Aging, 5(1), 119-126.

Henson, R. N. A., Hornberger, M., \& Rugg, M. D. (2005). Further dissociating the processes involved in recognition memory: An fMRI study. Journal of Cognitive Neuroscience, 17(7), 1058-1073. doi: 10.1162/0898929054475208

Herron, J. E., Henson, R. N. A., \& Rugg, M. D. (2004). Probability effects on the neural correlates of retrieval success: An fMRI study. NeuroImage, 21(1), 302-310. doi:10.1016/j.neuroimage.2003.09. 039

Hirshman, E. (1995). Decision processes in recognition memory: Criterion shifts and the list-strength paradigm. Journal of Experimental Psychology Learning, Memory, and Cognition, 21(2), 302-313.

Hutchinson, J. B., Uncapher, M. R., Weiner, K. S., Bressler, D. W., Silver, M. A., Preston, A. R., \& Wagner, A. D. (2014). Functional heterogeneity in posterior parietal cortex across attention and episodic memory retrieval. Cerebral Cortex 24(1), 49-66. doi:10.1093/ cercor/bhs 278

Jaeger, A., Konkel, A., \& Dobbins, I. G. (2013). Unexpected novelty and familiarity orienting responses in lateral parietal cortex during recognition judgment. Neuropsychologia, 51(6), 1061-1076. doi:10. 1016/j.neuropsychologia.2013.02.018

Johnson, M. K., Foley, M. A., Suengas, A. G., \& Raye, C. L. (1988). Phenomenal characteristics of memories for perceived and imagined autobiographical events. Journal of Experimental Psychology General, 117(4), 371-376.

Johnson, M. K., Raye, C. L., Foley, H. J., \& Foley, M. A. (1981). Cognitive operations and decision bias in reality monitoring. The American Journal of Psychology, 94(1), 37-64. doi:10.2307/ 1422342

Johnson, M. K., Raye, C. L., Foley, M. A., \& Kim, J. K. (1982). Pictures and images: Spatial and temporal information compared. Bulletin of the Psychonomic Society, 19(1), 23-26. doi:10.3758/BF03330029 
Johnson, J. D., \& Rugg, M. D. (2007). Recollection and the reinstatement of encoding-related cortical activity. Cerebral Cortex, 17(11), 25072515. doi:10.1093/cercor/bhl156

Kahn, I., Davachi, L., \& Wagner, A. D. (2004). Functionalneuroanatomic correlates of recollection: Implications for models of recognition memory. The Journal of Neuroscience, 24(17), 4172-4180. doi:10.1523/JNEUROSCI.0624-04.2004

Kim, H., \& Cabeza, R. (2007). Differential contributions of prefrontal, medial temporal, and sensory-perceptual regions to true and false memory formation. Cerebral Cortex, 17(9), 2143-2150. doi:10. 1093/cercor/bhl122

King, D. R., \& Miller, M. B. (2014). Lateral posterior parietal activity during source memory judgments of perceived and imagined events. Neuropsychologia, 53, 122-136. doi:10.1016/j.neuropsychologia. 2013.11.006

Klostermann, E. C., Loui, P., \& Shimamura, A. P. (2009). Activation of right parietal cortex during memory retrieval of nonlinguistic auditory stimuli. Cognitive, Affective, \& Behavioral Neuroscience, 9(3), 242-248. doi:10.3758/CABN.9.3.242

Kosslyn, S. M., Thompson, W. L., \& Alpert, N. M. (1997). Neural systems shared by visual imagery and visual perception: A positron emission tomography study. NeuroImage, 6(4), 320-334. doi:10. 1006/nimg.1997.0295

Lampinen, J. M., Odegard, T. N., \& Bullington, J. L. (2003). Qualities of memories for performed and imagined actions. Applied Cognitive Psychology, 17(8), 881-893. doi:10.1002/acp.916

Lancaster, J. L., Rainey, L. H., Summerlin, J. L., Freitas, C. S., Fox, P. T., Evans, A. C., ... Mazziotta, J. C. (1997). Automated labeling of the human brain: A preliminary report on the development and evaluation of a forward-transform method. Human Brain Mapping, 5(4), 238-242. doi:10.1002/(SICI)1097-0193(1997)5:43.0.CO;2-4

Lancaster, J. L., Woldorff, M. G., Parsons, L. M., Liotti, M., Freitas, C. S., Rainey, L., Kochunov, P. V., ... Fox, P. T. (2000). Automated Talairach atlas labels for functional brain mapping. Human Brain Mapping, 10(3), 120-131.

Leube, D. T., Erb, M., Grodd, W., Bartels, M., \& Kircher, T. T. J. (2003). Successful episodic memory retrieval of newly learned faces activates a left fronto-parietal network. Cognitive Brain Research, 18(1), 97-101. doi:10.1016/j.cogbrainres.2003.09.008

Levy, D. A. (2012). Towards an understanding of parietal mnemonic processes: Some conceptual guideposts. Frontiers in Integrative Neuroscience, 6, 41. doi:10.3389/fnint.2012.00041

Moritz, S., Gläscher, J., Sommer, T., Büchel, C., \& Braus, D. F. (2006). Neural correlates of memory confidence. NeuroImage, 33(4), 11881193. doi:10.1016/j.neuroimage.2006.08.003

Murnane, K., \& Bayen, U. J. (1996). An evaluation of empirical measures of source identification. Memory \& Cognition, 24(4), 417-428.

Nelson, S. M., Cohen, A. L., Power, J. D., Wig, G. S., Miezin, F. M., Wheeler, M. E., ... Petersen, S. E. (2010). A parcellation scheme for human left lateral parietal cortex. Neuron, 67(1), 156-170. doi:10. 1016/j.neuron.2010.05.025

Nyberg, L., Habib, R., McIntosh, A. R., \& Tulving, E. (2000). Reactivation of encoding-related brain activity during memory retrieval. Proceedings of the National Academy of Sciences of the United States of America, 97(20), 11120-11124.

Schooler, J. W., Gerhard, D., \& Loftus, E. F. (1986). Qualities of the unreal. Journal of Experimental Psychology Learning, Memory, and Cognition, 12(2), 171-181.

Shannon, B. J., \& Buckner, R. L. (2004). Functional-anatomic correlates of memory retrieval that suggest nontraditional processing roles for multiple distinct regions within posterior parietal cortex. The Journal of Neuroscience, 24(45), 10084-10092. doi:10.1523/ JNEUROSCI.2625-04.2004
Shimamura, A. P. (2011). Episodic retrieval and the cortical binding of relational activity. Cognitive, Affective, \& Behavioral Neuroscience, 11(3), 277-291. doi:10.3758/s13415-011-0031-4

Slotnick, S. D., Moo, L. R., Segal, J. B., \& Hart, J., Jr. (2003). Distinct prefrontal cortex activity associated with item memory and source memory for visual shapes. Cognitive Brain Research, 17(1), 75-82. doi:10.1016/S0926-6410(03)00082-X

Suengas, A. G., \& Johnson, M. K. (1988). Qualitative effects of rehearsal on memories for perceived and imagined complex events. Journal of Experimental Psychology: General, 117(4), 377-389. doi:10. 1037/0096-3445.117.4.377

Tomaiuolo, F., MacDonald, J. D., Caramanos, Z., Posner, G., Chiavaras, M., Evans, A. C., \& Petrides, M. (1999). Morphology, morphometry and probability mapping of the pars opercularis of the inferior frontal gyrus: An in vivo MRI analysis. The European Journal of Neuroscience, 11(9), 3033-3046.

Vaidya, C. J., Zhao, M., Desmond, J. E., \& Gabrieli, J. D. E. (2002). Evidence for cortical encoding specificity in episodic memory: Memory-induced re-activation of picture processing areas. Neuropsychologia, 40(12), 2136-2143. doi:10.1016/S00283932(02)00053-2

Vilberg, K. L., \& Rugg, M. D. (2007). Dissociation of the neural correlates of recognition memory according to familiarity, recollection, and amount of recollected information. Neuropsychologia, 45(10), 2216-2225. doi:10.1016/j.neuropsychologia.2007.02.027

Vilberg, K. L., \& Rugg, M. D. (2008). Memory retrieval and the parietal cortex: A review of evidence from a dual-process perspective. Neuropsychologia, 46(7), 1787-1799. doi:10.1016/j. neuropsychologia.2008.01.004

Vilberg, K. L., \& Rugg, M. D. (2009a). Functional significance of retrieval-related activity in lateral parietal cortex: Evidence from fMRI and ERPs. Human Brain Mapping, 30(5), 1490-1501. doi: 10.1002/hbm.20618

Vilberg, K. L., \& Rugg, M. D. (2009b). Left parietal cortex is modulated by amount of recollected verbal information. Neuroreport, 20(14), 1295-1299. doi:10.1097/WNR.0b013e3283306798

Wagner, A. D., Shannon, B. J., Kahn, I., \& Buckner, R. L. (2005). Parietal lobe contributions to episodic memory retrieval. Trends in Cognitive Sciences, 9(9), 445-453.

Weis, S., Klaver, P., Reul, J., Elger, C. E., \& Fernández, G. (2004). Temporal and cerebellar brain regions that support both declarative memory formation and retrieval. Cerebral Cortex, 14(3), 256-267. doi:10.1093/cercor/bhg125

Wernicke, C. (1874). Der aphasische Symptomencomplex, eine psychologische Studie auf anatomischer Basis, von Dr. C. Wernicke,... Breslau: M. Cohn und Weigert.

Wheeler, M. E., \& Buckner, R. L. (2003). Functional dissociation among components of remembering: Control, perceived oldness, and content. The Journal of Neuroscience: The Official Journal of the Society for Neuroscience, 23(9), 3869-3880.

Wheeler, M. E., Shulman, G. L., Buckner, R. L., Miezin, F. M., Velanova, K., \& Petersen, S. E. (2006). Evidence for separate perceptual reactivation and search processes during remembering. Cerebral Cortex, 16(7), 949-959. doi:10.1093/cercor/bhj037

Wilson, M. (1988). MRC psycholinguistic database: Machine-usable dictionary, version 2.00. Behavior Research Methods, Instruments, \& Computers, 20(1), 6-10. doi:10.3758/BF03202594

Woodruff, C. C., Johnson, J. D., Uncapher, M. R., \& Rugg, M. D. (2005). Content-specificity of the neural correlates of recollection. Neuropsychologia, 43(7), 1022-1032. doi:10.1016/j. neuropsychologia.2004.10.013 